\title{
EXACT SOLUTION OF THE PAIRING PROBLEM IN THE LST SCHEME
}

\author{
SING CHIN PANG ${ }^{\dagger}$ \\ Physics Department, University of Michigan, Ann Arbor, Michigan ${ }^{\dagger \dagger}$
}

Received 3 January 1969

\begin{abstract}
Exact solutions for the pairing interaction problem in the LST scheme are formulated in terms of the matrix elements of pair creation and annihilation operators for pairs coupled to zero orbital angular momentum. General expressions are given for these matrix elements for states with seniority $v=0$ and 1 . This makes it possible to study an orbital pairing interaction with different spin singlet and triplet strengths acting in mixed configurations of several single-particle levels. The mathematical formulation of the problem is based on the eight-dimensional quasispin group which is broken down according to the decomposition $\mathrm{O}(8) \supset \mathrm{O}(6) \supset[\mathrm{O}(3) \times \mathrm{O}(3)]$, where $O(6)$ corresponds to the usual Wigner supermultiplet symmetry group. A sample calculation for a simple two-level configuration, and states with $v_{1}=v_{2}=0$, nucleon numbers of $4,6,8,10$, shows (i) that the pairing interaction is very effective compared with the singleparticle excitations, and (ii) that it tends to make more stable those states built from the largest possible number of $\alpha$-like grouping of 4 particles.
\end{abstract}

\section{Introduction}

The quasispin method was first applied to problems in nuclear physics by Kerman ${ }^{1}$ ) in his treatment of the pairing interaction. Quasispin operators are built from pair creation and annihilation operators involving nucleon pairs coupled to zero angular momentum. In the $j-j$ coupling scheme of the shell model, involving configurations of identical nucleons (neutrons only or protons only), the quasispin operators have the commutation properties of conventional (three-dimensional) angular momentum operators. Generalization of the quasispin method to configurations with both neutrons and protons leads to operators of more complicated Lie algebras.

The quasispin group for fermions of arbitrary isospin was studied by Helmers ${ }^{2}$ ). The quasispin groups for configurations of both neutrons and protons in the conventional JT and LST schemes of nuclear physics have been identified by Flowers and Szpikowski ${ }^{3}$ ) and others ${ }^{4}$ ) as rotational groups in abstract spaces of 5 and 8 dimensions, respectively. In the LST scheme there are six pair creation operators coupled to orbital angular momentum $L=0$ (with spin and isospin $S=1, T=0$; or $S=0$, $T=1$ ) and a similar set of six pair annihilation operators. Flowers and Szpikowski have shown that these 12 operators together with the number operator and the 15 operators which are the $\mathrm{SU}(4)$ super multiplet operators of Wigner form the in-

+ Present address: Physics Dept., Univ. of Pennsylvania, Philadelphia, Pennsylvania.

${ }^{\dagger}+$ Supported by the US Office of Naval Research, Contract Nonr. 1224(59). 
finitesimal generators for an orthogonal group in eight dimensions. They have also evaluated the eigenvalue of the pairing Hamiltonian for the pure configuration $l^{N}$ in terms of the Casimir operators of $\mathrm{O}(8)$ and $\mathrm{SU}(4)$.

In this investigation matrix elements are evaluated for the $L=0$ pair creation and annihilation operators in the seniotity scheme in order to find exact solutions to the pairing problem in the LST scheme by matrix diagonalization techniques. This approach makes it possible to study the pairing interaction with different strengths for the $S=1(T=0)$ and $S=0(T=1)$ pairs, as well as for mixed configurations of several single-particle levels. It is thus possible to study the competition between pairing effects and single-particle excitations. Although the LST scheme may be a good zeroth approximation for some light nuclei, a two-body interaction approximated by a simple pairing interaction is not sufficient to describe the excitation spectra and binding energies of such nuclei. The present work is therefore intended mainly as a model study to further elucidate the properties of pairing interactions.

In the present work the group chain of $\mathrm{O}(8) \supset \mathrm{O}(7) \supset \mathrm{O}(6)$ is used, where $\mathrm{O}(6)$ can be identified with $\mathrm{SU}(4)$ and the representations of the Wigner supermultiplets, while the four numbers needed to specify the irreducible representations of the rank 4 group $O(8)$ can be identified with the quantum numbers of the seniority scheme. Although the irreducible representation labels of both $O(8)$ and $O(6)$ thus have ready physical significance, the quantum numbers of $O(7)$ have no easily identified physical meaning. Even worse, the nucleon number operator is in general not diagonal in a scheme based on the group chain $\mathrm{O}(8) \supset \mathrm{O}(7) \supset \mathrm{O}(6)$. To make the nucleon number, $N$, a good quantum number it is necessary to find specific linear combinations of the $\mathrm{O}(7)$ representations allowed by the $\mathrm{O}(8)$ and $\mathrm{O}(6)$ quantum numbers. A similar problem occurs when the Wigner supermultiplet representations of $\mathrm{O}(6)$ are further reduced to $S$ and $T$. In the canonical group chain $\mathrm{O}(6) \supset \mathrm{O}(5) \supset \mathrm{O}(4)$ $\supset \mathrm{O}(3) \supset \mathrm{O}(2)$ only one of the quantum numbers, either $S$ or $T$, can be identified with the irreducible representation of $\mathrm{O}(3)$. Because of these difficulties it has not been possible to give a completely general algebraic expression for the matrix elements of the pair operators, valid for all irreducible representations. However, if the seniority number $v$ is restricted to 0 or 1 the single quantum number $N$ is sufficient to completely specify the states of the $\mathrm{O}(8)$ to $\mathrm{O}(6)$ chain, while the states of the possible $\mathrm{O}(6)$ representations for these cases are fully identified by $S$ and $T$ only. Since the seniority $v$ gives the number of unpaired nucleons (entirely free of $L=0$ coupled pairs), states of lowest seniority such as $v=0$ and $v=1$ are precisely those of greatest interest in problems dominated by a pairing interaction.

In evaluating the matrix elements of the pair creation and annihilation operators it is useful to characterize these operators as irreducible tensors according to the group decomposition $\mathrm{O}(8) \supset \mathrm{O}(6) \supset[\mathrm{O}(3) \times \mathrm{O}(3)]$. This makes it possible to factor the matrix elements into separate pieces. The $\mathrm{O}(8) / \mathrm{O}(6)$ factor for states of seniority $v=0$ and 1 is evaluated in the present work. The $\mathrm{O}(6) /[\mathrm{O}(3) \times \mathrm{O}(3)]$ factor can be identified as a reduced Clebsch-Gordan coefficient for the Wigner supermultiplet 
scheme. These reduced $\mathrm{O}(6) /[\mathrm{O}(3) \times \mathrm{O}(3)]$, (or alternately $\mathrm{SU}(4) /[\mathrm{SU}(2) \times \mathrm{SU}(2)]$ ), coefficients will be used frequently in this paper. Tabulations of these coefficients will be given in a companion paper on SU(4) and the Wigner supermultiplet scheme ${ }^{8}$ ). Finally, since the two sets of commuting $O(3)$ generators correspond to the spin and isospin operators, the two $\mathrm{O}(3) / \mathrm{O}(2)$ factors for the matrix elements of the pair operators are ordinary spin and isospin angular momentum coupling coefficients which carry the dependence on $M_{S}$ and $M_{T}$.

In sect. 2 the properties of $O(8)$ and $O(6)$ are reviewed. The correspondence is established between the quasispin operators and the infinitesimal generators of the orthogonal groups; the ways of characterizing the irreducible representations of $\mathrm{O}(8)$ and $\mathrm{O}(6)$ are reviewed; and the irreducible tensor classification of the pair operators is given. In sect. 3 the $\mathrm{O}(8) / \mathrm{O}(6)$ factors for the matrix elements of the pair operators are evaluated for states with $v=0$ and 1 . To accomplish this the transformation must first be made from the $\mathrm{O}(8) \supset \mathrm{O}(7) \supset \mathrm{O}(6)$ scheme to one in which the nucleon number operator, $N$, is diagonal. In sect. 4 the applications to the pairing problem are given. The effect of a pairing interaction with different strenghts for the $(S=1$, $T=0)$ and $(S=0, T=1)$ pairs is discussed in detail for the case of one isolated single-particle level. The techniques for calculating the matrix elements of the most general pairing Hamiltonian for a nuclear system with many single-particle levels are developed. Finally, results of a sample calculation are given for a system with a single-particle spectrum similar to that of the s-d shell and a pairing interaction which (for convenience of analysis) is assumed to have state and spin independent strength. The needed diagonalizations have been done numerically by computer; 1esults are shown for nucleon numbers of $4,6,8$ and 10 .

An alternate technique for finding solutions for the eigenvalues of a charge, spin independent pairing Hamiltonian has recently been given by Richardson ${ }^{5}$ ). However, Richardson's approach is very different from the present one. It requires the solution of a system of coupled algebraic equations with subsidiary conditions. With general expressions for the matrix elements of the pair operators, the present method (as emphasized before) can treat the case with different spin triplet and singlet pairing strengths and makes it easier to consider perturbation treatments for the weak or strong pairing limits.

\section{The eight-dimensional quasispin group}

In an LST scheme the quasispin operators are built from the pair operators which create or annihilate pairs of nucleons coupled to total orbital angular momentum, $L=0$. These can be constructed from the creation and annihilation operators for single nucleons which are characterized by the single-particle spatial quantum numbers $(n l m)$; and $s m_{s}, t m_{t}$; (with $s=\frac{1}{2}, t=\frac{1}{2}, m_{t}=+\frac{1}{2}\left(-\frac{1}{2}\right)$ for neutron (and proton), respectively). The operators 


$$
\begin{aligned}
2 S+1,2 T+1 A^{\dagger}\left(M_{S}, M_{T}\right) & =\sum_{\substack{m_{s}, m_{t}, m \\
(n, l)}} \sqrt{l+\frac{1}{2}}\langle l m l-m \mid 00\rangle\left\langle\frac{1}{2} m_{t} \frac{1}{2} m_{t}^{\prime} \mid T M_{T}\right\rangle \\
& \times\left\langle\frac{1}{2} m_{s} \frac{1}{2} m_{s}^{\prime} \mid S M_{S}\right\rangle a_{n l m m_{s} m_{t}}^{\dagger} a_{n l-m m_{s}^{\prime} m_{t}^{\prime},}^{\dagger} \\
& 2 S+1,2 T+1+{ }^{1} A\left(M_{S}, M_{T}\right)=\left({ }^{2 S+1,2 T+1} A^{\dagger}\left(M_{S}, M_{T}\right)\right)^{\dagger},
\end{aligned}
$$

are, in addition, coupled to total spin and isospin, $S$ and $T$; where, with $L=0$, the anticommutation properties of $a^{+}$restrict the $S T$ values to be either 10 or 01 . The commutators of $A^{\dagger}$ with $A$ lead to 16 number preserving operators. These include the number operator, which appears naturally in the combination

$$
Q_{0}=\left(-\frac{1}{2} N_{\mathrm{op}}+\Omega\right)
$$

where $\Omega=$ spatial degeneracy number; (e.g. $\Omega=2 l+1$ for a single level, and $\Omega=\sum_{a}\left(2 l_{a}+1\right)$ for a set of degenerate single-particle levels of orbital angular momenta $l_{a}$ ). The remaining number preserving operators are the three components of the spin and isospin operators $S$ and $T$, and the 9 components of the operator $E$

$$
E_{a b}=\sum_{(n l) m m_{s} m_{t}}\left\langle\left(m_{s}+a\right)\left(m_{t}+b\right)\left|\sigma_{a} \tau_{b}\right| m_{s} m_{t}\right\rangle a_{n l m\left(m_{s}+a\right)\left(m_{t}+b\right)}^{\dagger} a_{n l m m_{s} m_{t}}
$$

where $\sigma_{a}$ and $\tau_{b}$ are spherical components of the single-particle Pauli spin and isospin operators. The 15 operators $\boldsymbol{S}, \boldsymbol{T}$, and $\boldsymbol{E}$ are the Wigner supermultiplet operators which generate the group $\mathrm{SU}(4)$, or alternately an orthogonal group in dimensions.

The full set of 28 quasispin operators $A^{+}, A, Q_{0}, \boldsymbol{S}, \boldsymbol{T}$ and $\boldsymbol{E}$ are the infinitesimal operators which generate the group $\mathrm{O}(8)$. The detailed correspondence between the quasispin operators and the generators of $\mathrm{O}(8)$ has been given by Flowers and Szpikowski ${ }^{3}$ ). It is shown in table 1. Besides the four self-adjoint operators $Q_{0}, S_{0}$, $T_{0}$, and $E_{00}$, only 12 other operators are shown explicitly, since the remaining 12 can be obtained from these by hermitian conjugation.

The $n$ dimensional orthogonal groups are generated by a set of $\frac{1}{2} n(n-1)$ operators $J_{\alpha \beta}$. These generators are governed by the commutation rule

$$
\begin{aligned}
& {\left[J_{p q}, J_{r s}\right]=i\left(\delta_{s p} J_{r q}+\delta_{r q} J_{s p}-\delta_{r p} J_{s q}-\delta_{s q} J_{r p}\right),} \\
& J_{p q}=-J_{q p}, \quad p, q=1,2, \ldots, n . \\
& J_{p q}^{\dagger}=J_{p q},
\end{aligned}
$$

The rank of the group, which is the number of mutually commuting operators and also the number of integers or half-integers required to specify the irreducible representations, is $k$ for $n=2 k$ and $n=2 k+1$. For example, 4 numbers are required to specify the irreducible representation of $O(8)$, where as only 3 are needed for both $O(6)$ and $\mathrm{O}(7)$.

To completely specify the states of a given irreducible representation of $\mathrm{O}(n)$, a set of $\frac{1}{2}\left(\frac{1}{2} n(n-1)-k\right)$ additional quantum numbers or commuting operators are 
TABLE 1

Infinitesimal operators of $O(8)$

a)

Quasispin operators b) c)

Eight dimensional "angular momentum" operators "angular momentum" d)

Flowers-Szpikowski

\begin{tabular}{|c|c|c|c|c|}
\hline${ }^{31} A^{\dagger}(1,0)$ & $Q_{+}^{\text {np }}$ & {$\left[-\left(J_{17}+i J_{27}\right)+i\left(J_{18}+i J_{28}\right)\right]$} & 1 & 0 \\
\hline${ }^{31} A^{\dagger}(0,0)$ & $\frac{1}{\sqrt{ } 2}\left(Q_{+}^{\mathrm{n}-\mathrm{p}}+Q_{+}^{-\mathrm{np}}\right)$ & $\frac{-i\left(J_{37}-i J_{38}\right)}{\sqrt{ } 2}$ & 1 & 0 \\
\hline${ }^{31} A^{\dagger}(-1,0)$ & $Q_{+}^{-\mathrm{n}-\mathrm{p}}$ & $\frac{1}{2}\left[-\left(J_{17}-i J_{27}\right)+i\left(J_{18}-i J_{28}\right)\right]$ & 1 & 0 \\
\hline${ }^{13} A^{\dagger}(0,1)$ & $Q_{+}^{n-n}$ & $\frac{1}{2}\left[i\left(J_{57}+i J_{67}\right)+\left(J_{58}+i J_{68}\right)\right]$ & 1 & 0 \\
\hline${ }^{13} A^{\dagger}(0,0)$ & $\frac{1}{\sqrt{ } 2}\left(Q_{+}^{\mathrm{p}-\mathrm{n}}+Q_{+}^{\mathrm{n}-\mathrm{p}}\right)$ & $\frac{\left(J_{47}-i J_{48}\right)}{\sqrt{ } 2}$ & 1 & 0 \\
\hline${ }^{13} A^{\dagger}(0,-1)$ & $Q_{+}^{\mathrm{p}-\mathrm{p}}$ & $\frac{1}{2}\left[i\left(J_{57}-i J_{67}\right)+\left(J_{58}-i J_{68}\right)\right]$ & 1 & 0 \\
\hline$Q_{0}=\Omega-\frac{1}{2} N$ & $-\frac{1}{2}\left(Q_{0}^{\mathrm{nn}}+Q_{0}^{\mathrm{pp}}+Q_{0}^{-\mathrm{n}-\mathrm{n}}+Q_{0}^{-\mathrm{p}-\mathrm{p}}\right)+\Omega$ & $J_{78}$ & 0 & 0 \\
\hline$S_{0}$ & $\frac{1}{2}\left(Q_{0}^{\mathrm{nn}}+Q_{0}^{\mathrm{pp}}-Q_{0}^{-\mathrm{n}-\mathrm{n}}-Q_{0}^{-\mathrm{p}-\mathrm{p}}\right)$ & $J_{12}$ & 1 & 1 \\
\hline$T_{0}$ & $\frac{1}{2}\left(Q_{0}^{\mathrm{nn}}-Q_{0}^{\mathrm{pp}}+Q_{0}^{-\mathrm{n}-\mathrm{n}}-Q_{0}^{-\mathrm{p}-\mathrm{p}}\right)$ & $J_{56}$ & 1 & 1 \\
\hline$E_{00}$ & $\frac{1}{2}\left(Q_{0}^{\mathrm{nn}}-Q_{0}^{\mathrm{pp}}-Q_{0}^{-\mathrm{n}-\mathrm{n}}+Q_{0}^{-\mathrm{p}-\mathrm{p}}\right)$ & $J_{34}$ & 1 & 1 \\
\hline$S_{+}$ & $\frac{1}{\sqrt{ } 2}\left(Q_{0}^{\mathrm{n}-\mathrm{n}}+Q_{0}^{\mathrm{p}-\mathrm{p}}\right)$ & $\frac{1}{\sqrt{2}}\left(J_{13}+i J_{23}\right)$ & 1 & 1 \\
\hline$T_{+}$ & $\frac{1}{\sqrt{ } 2}\left(Q_{0}^{\mathrm{np}}+Q_{0}^{-\mathrm{n}-\mathrm{p}}\right)$ & $\frac{1}{\sqrt{2}}\left(J_{45}+i J_{46}\right)$ & 1 & 1 \\
\hline$E_{11}$ & $Q_{0}^{\mathrm{n}-\mathrm{p}}$ & $\frac{1}{2}\left[\left(J_{15}+i J_{25}\right)+i\left(J_{16}+i J_{26}\right)\right]$ & 1 & 1 \\
\hline$E_{1-1}$ & $Q_{0}^{\mathrm{p}-\mathrm{n}}$ & $\frac{1}{2}\left[-\left(J_{15}+i J_{25}\right)+i\left(J_{16}+i J_{26}\right)\right]$ & 1 & 1 \\
\hline$E_{10}$ & $\frac{1}{\sqrt{ } 2}\left(Q_{0}^{\mathrm{n}-\mathrm{n}}-Q_{0}^{\mathrm{p}-\mathrm{p}}\right)$ & $\frac{i}{\sqrt{2}}\left(J_{14}+i J_{24}\right)$ & 1 & 1 \\
\hline$E_{01}$ & $\frac{1}{\sqrt{2}}\left(Q_{0}^{\mathrm{np}}-Q_{0}^{-\mathrm{n}-\mathrm{p}}\right)$ & $\frac{-i}{\sqrt{2}}\left(J_{35}+i J_{36}\right)$ & 1 & 1 \\
\hline
\end{tabular}

a) The quasispin operators are defined by eqs. (2.1)-(2.4). Note that $E_{-a,-b}=E_{a b}^{+}, S_{-}=S_{+}^{+}, T_{-}=T_{+}^{+}$.

b) $Q_{+}^{\alpha \beta}=\Sigma_{m}(-1)^{l-m} a_{n l m-\alpha}^{+} a_{n l-m \beta}^{+}, \quad Q_{-}^{\beta \alpha}=\left(Q_{+}^{\alpha \beta}\right)^{+}, \quad Q_{o}^{\alpha \beta}=\Sigma_{m} a_{n l m \alpha}^{+} a_{n l m \beta}$,

where $\alpha$ and $\beta$ are specified by $m_{s}$ and $m_{t}$ (e.g., $\alpha=-p$ stands for a "spin down" proton). For several degenerate single-particle levels the sum over $m$ is replaced by a sum over $n l m$.

c) The eight-dimensional "angular momentum" operators satisfy eqs. (2.5). The vectors $\boldsymbol{S}$ and $\boldsymbol{T}$ have been chosen to span the $1,2,3$ and $4,5,6$, subspaces respectively.

d) $\mathbf{O ( 6 )}$ irreducible tensor character of the operators. 
needed. The physics of the applications generally forces specific choices for certain of these operators. The mathematically most natural way to completely specify the state vectors, however, is given by the Weyl, Gelfand-Zetlin scheme, based on the canonical chain of subgroups $\mathrm{O}(n) \supset \mathrm{O}(n-1) \supset \mathrm{O}(n-2) \supset \ldots . \mathrm{O}(2)$; where the $\frac{1}{2}\left(\frac{1}{2} n(n-1)-k\right)$ quantum numbers are given by the labels which specify the irreducible representations of all the subgroups in the chain. For $O(8)$ a basis vector in this scheme is characterized by the Gelfand pattern

$$
\left|\begin{array}{llll}
m_{81} & m_{82} & m_{83} & m_{84} \\
m_{71} & m_{72} & m_{73} & \\
m_{61} & m_{62} & m_{63} \\
m_{51} & m_{52} & \\
m_{41} & m_{42} & \\
m_{31} & & \\
m_{21} & &
\end{array}\right|
$$

where $m_{n i}$ are the irreducible representation labels for the subgroup $\mathrm{O}(n)$. E. g., $m_{61}$, $m_{62}, m_{63}$ are the three numbers needed to specify the irreducible representations of $\mathrm{O}(6)$. The irreducible representation labels $m_{n i}$ satisfy the branching rule ${ }^{6}$ ):

$$
m_{n+1, i} \geqq m_{n i} \geqq m_{n+1, i+1} \text {. }
$$

The integers or half-integers $m_{n i}$ are positive with the exceptions of $m_{2 k, k}$ which can be both positive and negative. (In the branching rule $m_{2 k, k}$ must be replaced by its absolute value.)

In the above basis for $\mathrm{O}(8)$, however, the number operator and the isospin operators $T^{2}$ and $T_{0}$ are in general not diagonal ${ }^{\dagger}$. The Gelfand basis is therefore not the physically relevant one for the pairing problem of nuclear physics. However, since the matrix elements of $J_{\alpha \beta}$ are known in the Gelfand basis ${ }^{6}$ ), we shall often return to the scheme (2.6).

Although the full Gelfand state vector is not directly useful for the physical applications, the $\mathrm{O}(6)$ and $\mathrm{O}(8)$ irreducible representation labels $m_{6 i}$ and $m_{8 i}$ can be related to the Wigner supermultiplet and seniority quantum numbers, respectively.

\subsection{THE IRREDUCIBLE REPRESENTATIONS OF O(6)}

The irreducible representations of $\mathrm{O}(6)$ are specified by the highest weights, that is by the largest possible eigenvalues of the 3 commuting operators $S_{0}, E_{00}, T_{0}$. Since $O(6)$ and $S U(4)$ have Lie algebras of the same structure, the irreducible representations can also be labeled according to the notation standard for the special unitary group associated with the four-dimensional spin-isospin space. It is convenient to include the number operator (or $Q_{0}$ ) which commutes with the 15 operators

+ By the specific choice of operators made in the present work, (see column (c) of table 1), the quantum numbers $S M_{S}$ correspond to $m_{31} m_{21}$. 
$S, T, E_{\alpha \beta}$, and together with them generates a group U(4), so that the irreducible representations can be specified by the symmetries of $n$-nucleon spin-isospin functions. These symmetries are characterized by Young tableaus or partition numbers $\left[f_{1} f_{2} f_{3} f_{4}\right]$ on $N$ objects where $f_{i}$ are integers such that $\left(f_{1}+f_{2}+f_{3}+f_{4}=N\right)$ with $\Omega \geqq f_{1} \geqq f_{2} \geqq f_{3} \geqq f_{4} \geqq 0$. The partition number $f_{i}$ specifies the length of the $i$ th row of the Young tableau. In a spin, isospin function with symmetry characterized by the $f_{i}$, there can be at most $f_{1}$ neutrons with spin up, and subject to this restriction at most $f_{2}$ additional protons with spin up etc..... The largest possible eigenvalues of the 3 commuting operators $S_{0}, E_{00}, T_{0}$ are thus specified by the partition numbers $f_{i}$. Independent of the ordering of the single-particle states. The highest weights which define the $\mathrm{O}(6)$ irreducible representations are thus characterized by

$$
\begin{aligned}
& P=\frac{1}{2}\left(f_{1}+f_{2}-f_{3}-f_{4}\right), \\
& P^{\prime}=\frac{1}{2}\left(f_{1}-f_{2}+f_{3}-f_{4}\right), \\
& P^{\prime \prime}=\frac{1}{2}\left(f_{1}-f_{2}-f_{3}+f_{4}\right) .
\end{aligned}
$$

These $P, P^{\prime}, P^{\prime \prime}$ are the Wigner supermultiplet quantum numbers. They also correspond to the standard labels of the Gelfand scheme, that is $\left(P P^{\prime} P^{\prime \prime}\right) \equiv\left(m_{61} m_{62} m_{63}\right)$. To avoid confusion in the following, standard $\mathrm{O}(6)$ labels will always be enclosed by round parentheses, standard $\mathrm{U}(4)$ or $\mathrm{SU}(4)$ labels by square brackets, (the latter enclosing 4 or 3 numbers for $\mathrm{U}(4)$ or $\mathrm{SU}(4)$, respectively).

\subsection{THE IRREDUCIBLE REPRESENTATIONS OF O(8)}

The irreducible representations of $\mathrm{O}(8)$ are specified by the highest weights, defined by the largest possible eigenvalues of the 4 commuting operators $Q_{0}, T_{0}, E_{00}, S_{0}$. In a state of specified seniority $v$, there must be at least $v$ nucleons (the number entirely free of pairs coupled to $L=0$ ). The highest possible eigenvalues of the generator $Q_{0}=J_{78}=\Omega-\frac{1}{2} N_{\mathrm{op}}$ is therefore

$$
Q=\Omega-\frac{1}{2} v .
$$

Subject to the restriction to the highest possible $Q_{0}$, the highest eigenvalues of $S_{0}, E_{00}$, and $T_{0}$ are therefore specified by the symmetry of a $v$ nucleon spin-isospin function characterized by partition numbers $\left[\mu_{1} \mu_{2} \mu_{3} \mu_{4}\right]$ on $v$ objects, where $\mu_{1}+\mu_{2}+\mu_{3}+\mu_{4}=v$ and where the $\mu_{i}$ are again integers, satisfying

$$
\frac{1}{2} \Omega \geqq \mu_{1} \geqq \mu_{2} \geqq \mu_{3} \geqq \mu_{4} \leqq 0 .
$$

Among the set of $v$ nucleons corresponding to the highest weight state there can again be at most $\mu_{1}$ neutrons with spin up, and subject to this restriction at most $\mu_{2}$ additional protons with spin up, etc.... The highest eigenvalues of $S_{0}, E_{00}, T_{0}$ in the highest weight state of $\mathrm{O}(8)$ are therefore specified by

$$
\begin{aligned}
& p=\frac{1}{2}\left(\mu_{1}+\mu_{2}-\mu_{3}-\mu_{4}\right), \\
& p^{\prime}=\frac{1}{2}\left(\mu_{1}-\mu_{2}+\mu_{3}-\mu_{4}\right), \\
& p^{\prime \prime}=\frac{1}{2}\left(\mu_{1}-\mu_{2}-\mu_{3}+\mu_{4}\right),
\end{aligned}
$$


and in standard Gelfand form the $\mathrm{O}(8)$ irreducible representations are specified by $\left(m_{81} m_{82} m_{83} m_{84}\right)=\left(\Omega-\frac{1}{2} v, p p^{\prime} p^{\prime \prime}\right)$.

The $p, p^{\prime}, p^{\prime \prime}$ are called reduced supermultiplet quantum numbers. They are the supermultiplet quantum numbers of the $v$ nucleons free of pairs coupled to $L=0$. The highest weight state with $N=v$ nucleons must satisfy the relation

$$
{ }^{2 S+1,2 T+1} A\left(M_{S}, M_{T}\right)\left|N=v, p p^{\prime} p^{\prime \prime}\right\rangle=0 .
$$

The remaining states of the $\mathrm{O}(8)$ irreducible representation characterized by $v, p, p^{\prime} p^{\prime \prime}$ corresponding to nucleon number $N$ are obtained by acting on the state $\left|N=v, p p^{\prime} p^{\prime \prime}\right\rangle$ with $\frac{1}{2}(N-v)$ operators $A^{+}$.

\subsection{DECOMPOSITION OF O(8) INTO O(6)}

A specific $\mathrm{O}(8)$ irreducible representation denoted by $\left(\Omega-\frac{1}{2} v, p, p^{\prime}, p^{\prime \prime}\right)$, decomposes into different irreducible representations of the subgroup $\mathrm{O}(6)$ characterized by $\left(P, P^{\prime}, P^{\prime \prime}\right)$. This decomposition is due to the fact that the $\frac{1}{2}(N-v)$ operators $A^{+}$ with $\mathrm{O}(6)$ irreducible tensor character $(100)$ can be coupled to the $\mathrm{O}(6)$ representations $\left(p, p^{\prime}, p^{\prime \prime}\right)$ in many different ways.

For fixed $\left(\Omega-\frac{1}{2} v, p, p^{\prime}, p^{\prime \prime}\right)$ and $\left(P, P^{\prime}, P^{\prime \prime}\right)$ the decomposition from $\mathrm{O}(8)$ to $\mathrm{O}(6)$ is not unique. For the $\mathrm{O}(8) \supset \mathrm{O}(7) \supset \mathrm{O}(6)$ chain it can be seen that three other quantum numbers which specify the representations of $O(7)$ are needed to identify the "parent" of $\mathrm{O}(6)$. Unfortunately these three quantum numbers have no easily determined physical content.

A similar problem occurs in the decomposition of $\mathrm{O}(6)$ into the direct product $[\mathrm{O}(3) \times \mathrm{O}(3)]$ of the spin and isospin groups. Once again the set of $\left(P, P^{\prime}, P^{\prime \prime}\right)$ and $\left(S M_{S}, T M_{T}\right)$ are not sufficient to give a unique decomposition. Two other quantum numbers are needed to completely specify a state. Although Moshinsky and Nagel ${ }^{7}$ ) have succeeded in finding the needed operators, they are of third and fourth degree in the infinitesmal operators of $\mathrm{O}(6)$ and the physical content of these two operators is again obscure.

The present work tries to do without these additional quantum numbers at the cost of limiting the scope of interest to include only states of low seniority. Since these are precisely the states of greatest interest for problems in pairing theory, this is not a severe restriction.

In the most general case a state vector for a specific $\mathrm{O}(8)$ irreducible representation would be completely specified by 16 quantum numbers

$$
\left|\left(Q=\Omega-\frac{1}{2} v, p, p^{\prime}, p^{\prime \prime}\right),\left(m_{71} m_{72} m_{73}\right),\left(P P^{\prime} P^{\prime \prime}\right), S M_{S} T M_{T}, \omega \phi\right\rangle .
$$

Where, for example, $\omega$ and $\phi$ could be chosen as the eigenvalues of Moshinsky and Nagel's third and fourth degree $\mathrm{O}(6)$ operators, while $\left(m_{71} m_{72} m_{73}\right)$ are the representation labels of the group $O(7)$ in the group chain $O(8) \supset O(7) \supset O(6)$. The 
general branching law, eq. (2.7), requires

$$
\begin{aligned}
& Q \geqq m_{71} \geqq p, \quad p \geqq m_{72} \geqq p^{\prime}, \quad p^{\prime} \geqq m_{73} \geqq p^{\prime \prime}, \\
& m_{71} \geqq P, \quad P \geqq m_{72} \geqq P^{\prime}, \quad P^{\prime} \geqq m_{73} \geqq\left|P^{\prime \prime}\right| \text {. }
\end{aligned}
$$

If the seniority $v$ is restricted to be either 0 or $1, p p^{\prime} p^{\prime \prime}$ are restricted to the values 000 or $\frac{1}{2} \frac{1}{2} \frac{1}{2}$, respectively. In these special cases the remaining quantum numbers are therefore also severely restricted as indicated in table 2.

TABLE 2

\begin{tabular}{lll} 
& For $v=0$ & \multicolumn{1}{c}{ For $v=1$} \\
\cline { 2 - 3 } $\mathrm{O}(8)=\left(Q p p^{\prime} p^{\prime \prime}\right)$ & $(\Omega 000)$ & $\left(\Omega-\frac{1}{2}, \frac{1}{2} \frac{1}{2} \frac{1}{2}\right)$ \\
$\mathrm{O}(7)=\left(m_{71} m_{72} n_{73}\right)$ & $(m 00)$ & $\left(m^{\prime} \frac{1}{2} \frac{1}{2}\right)$ \\
$\mathrm{O}(6)=\left(P P^{\prime} P^{\prime \prime}\right)$ & $(n 00)$ & $\left(n-\frac{1}{2}, \frac{1}{2}, \pm \frac{1}{2}\right)$ \\
& $m=$ integer & $m^{\prime}=\frac{1}{2}$-integer \\
& $\Omega \geqq m \geqq n$ & $\Omega-\frac{1}{2} \geqq m^{\prime} \geqq n-\frac{1}{2}$ \\
\hline
\end{tabular}

In both cases therefore only a single $O(7)$ quantum number is needed to completely specify the states. However, the quantum numbers $m$ have no ready physical significance. Even worse, a state of definite $m$ is not a state of a definite number of nucleons, $N$. The number operator is in general not diagonal in the $\mathrm{O}(8) \supset \mathrm{O}(7) \supset \mathrm{O}(6)$ scheme. (The highest weight state is an exception.) Since it can be shown that the number of distinct eigenvalues of the number operator is equal to $\Omega-n+1$, which is equal to the number of distinct values of $m$ (or $m^{\prime}$ ) in the above two cases, the number operator itself can be used, in place of an operator whose eigenvalues determine $m$, as the additional operator which makes the decomposition of $O(8)$ into $O(6)$ unique in these two cases. Restriction of $v$ to either 0 or 1 implies a restriction to the $O(6)$ representations $(n 00)$ or $\left(n-\frac{1}{2}, \frac{1}{2} \frac{1}{2}\right)$ and $\left(n-\frac{1}{2}, \frac{1}{2},-\frac{1}{2}\right)$. It has been shown $\left.{ }^{8}\right)$ that the decomposition of $\mathrm{O}(6)$ into $[\mathrm{O}(3) \times \mathrm{O}(3)]$ is unique in these special cases, so that the quantum numbers $S M_{S} T M_{T}$ are sufficient to completely specify the states of $\mathrm{O}(6)$.

The matrix elements of the pair operators are to be calculated by a generalized Wigner-Eckart theorem so that it is important to characterize these operators as irreducible tensors according to the group decomposition $\mathrm{O}(8) \supset \mathrm{O}(6) \supset[\mathrm{O}(3) \times$ $\times \mathrm{O}(3)$ ]. The 28 infinitesimal operators transform according to the regular representation (1100) of $O(8)$. A single $O(7)$ label again suffices to specify the states in this representation. In place of the $\mathrm{O}(7)$ label, however, the operators can be characterized by the change $\Delta N$ which they induce in the nucleon number. In place of $\Delta N$ it will be convenient to use $\Delta \lambda$ where $\lambda$ is the eigenvalue of the operator $\left(\Omega-\frac{1}{2} N_{\mathrm{op}}\right)$. Obviously, $\Delta N= \pm 2,0$; or $\Delta \lambda=\mp 1,0$; for $A^{\dagger}, A$, and the number preserving operators, respectively. Since the operators $A^{\dagger},(A)$ create (or annihilate) a pair of nucleons with antisymmetric spin, isospin functions, their SU(4) tensor character is [110] 
which corresponds to the self-conjugate representation (100) of $\mathrm{O}(6)$. Finally, the 15 infinitesimal operators $S, T$, and $E$ which generate $\mathrm{O}(6)$ transform according to the regular representation (110), while the operator $Q_{0}$ is a scalar, $\mathrm{O}(6)$ representation $(000)$. In all of these $\mathrm{O}(6)$ representations the quantum numbers $S M_{S}, T M_{T}$ are sufficient. The irreducible tensors can thus be characterized by $T_{\Delta \lambda,\left(P P^{\prime} P^{\prime \prime}\right)}^{(1100)}, S M_{S}, T M_{T}$. The $\mathrm{O}(6)$ character is indicated in table 1 . The $S M_{S}, T M_{T}$ character follows directly from the notation. In particular

$$
{ }^{2 S+1,2 T+1} A^{+}\left(M_{S}, M_{T}\right)=T_{-1,(100), S M S, T M_{T}}^{(1100)} .
$$

\section{The $\mathbf{O}(8) / O(6)$ part of the pairing problem}

In order to find solutions to the pairing problem, the matrix elements of the pair creation and annihilation operators are to be evaluated. By the tensor classification according to $\mathrm{O}(8) \supset \mathrm{O}(6) \supset[\mathrm{O}(3) \times \mathrm{O}(3)]$ these can be factored into segments. The $\mathrm{O}(8) / \mathrm{O}(6)$ segment is to be evaluated in this section. The $\mathrm{O}(6) \supset[\mathrm{O}(3) \times \mathrm{O}(3)]$ segment can be identified as a reduced Wigner supermultiplet Clebsch-Gordan coefficient. These $\mathrm{O}(6) \supset[\mathrm{O}(3) \times \mathrm{O}(3)]$ coefficients are calculated and tabulated in a companion paper on the Wigner supermultiplet scheme. ${ }^{8}$ )

To calculate the $\mathrm{O}(8) / \mathrm{O}(6)$ factors of the matrix elements it is first necessary to make a transformation to a basis in which the number operator is diagonal. This physically meaningful basis is constructed as a linear combination of the basis vectors for the mathematically natural or canonical group chain $O(8) \supset O(7) \supset O(6)$. For the case of states with seniority $v=0$ or 1 , the requirement that nucleon number $N$ be a good quantum number is sufficient to determine the coefficients in this expansion. The details for the case $v=0$ will be shown in subsect. 3.1. The calculations for $v=1$ are similar (see also ref. ${ }^{9}$ )). The technique used for the calculation of the matrix elements of the pair operators is again illustrated in detail only for the case $v=0$, in sect. 3.2. Results for the case $v=1$ are given in subsect. 3.3.

\subsection{THE BASIS WHICH DIAGONALIZES THE NUMBER OPERATOR}

For $v=0$, the $\mathrm{O}(8)$ representations are characterized by $(\Omega 000)$ and the possible $\mathrm{O}(6)$ representations by $(n 00)$. The possible values of $N$ (nucleon number) for fixed $\Omega$ and $n$ can be determined by counting the number of nodes in the possible Young tableau for $(n 00)$, that is $\mathrm{SU}(4)$ representation [ $n n 0$ ]. These Young tableaus must consist of $n$ columns of 2 nodes and a possible additional set of $i$ columns of 4 nodes, where $i=0,1,2, \ldots, \Omega-n$. (The maximum number of columns in the tableau is limited by $\Omega$, the spatial degeneracy.) The possible values of $N$ are thus given by $N=2 n+4 i$. The corresponding eigenvalues of $Q_{0}$ are $\lambda=\Omega-n-2 i$.

The $\mathrm{O}(7)$ representations are characterized by $(m 00)$ where $n \leqq m \leqq \Omega$. There are altogether $(\Omega-n+1)$ different values of $m$. On the other hand, $Q_{0}$ or $J_{78}$ have just $(\Omega-n+1)$ distinct eigenvalues, so that nucleon number can be used in place of $m$. 
A state vector in the $\mathrm{O}(8) \supset \mathrm{O}(7) \supset \mathrm{O}(6)$ basis can be written in an abbreviated form of eq. (2.6) by

$$
\varphi_{\Omega m n}=\left|\begin{array}{cccc}
\Omega & 0 & 0 & 0 \\
m & 0 & 0 & \\
n & 0 & 0 & \\
\vdots & \vdots & &
\end{array}\right|
$$

where all the quantum numbers that specify the subspace of $O(6)$ are omitted. (They can have any arbitrary value.) An eigenbasis for the number operator is given by the expansion

$$
\Psi_{\Omega n}^{(\lambda)}=\sum_{m=n}^{\Omega} D_{m n}^{(\lambda)} \varphi_{\Omega m n}
$$

which must satisfy

$$
J_{78} \Psi_{\Omega n}^{(\lambda)}=\lambda_{\Omega n} \Psi_{\Omega n}^{(\lambda)}
$$

where

$$
\lambda_{\Omega n}=\Omega-\frac{1}{2} N=(\Omega-n)-2 i, \quad i=0,1,2, \ldots,(\Omega-n) .
$$

The matrix elements of $J_{k-1 k}$ in the canonical basis are known. ${ }^{6}$ ) Operating with $J_{78}$ on a state vector of the canonical basis $\mathrm{O}(8) \supset \mathrm{O}(7) \supset \mathrm{O}(6)$ gives

$$
J_{78} \varphi_{\Omega m n}=A_{\Omega m n+} \varphi_{\Omega m+1 n}+A_{\Omega m n}-\varphi_{\Omega m-1 n},
$$

where the coefficients are

$$
\begin{gathered}
A_{\Omega m n+}=-i\left[\frac{(\Omega-m)(\Omega+m+6)(m+n+5)(m-n+1)}{(2 m+5)(2 m+7)}\right]^{\frac{1}{2}}, \\
A_{\Omega m n-}=i\left[\frac{(\Omega-m+1)(\Omega+m+5)(m+n+4)(m-n)}{(2 m+3)(2 m+5)}\right]^{\frac{1}{2}} .
\end{gathered}
$$

From eqs. (3.2), (3.3) and (3.4) we get the recursion relations

$$
\begin{aligned}
& D_{m-1 n}^{(\lambda)}\left[\frac{(m+n+4)(m-n)(\Omega+m+5)(\Omega-m+1)}{(2 m+3)(2 m+5)}\right]^{\frac{1}{2}} \\
= & D_{m+1 n}^{(\lambda)}\left[\frac{(m+n+5)(m-n+1)(\Omega+m+6)(\Omega-m)}{(2 m+5)(2 m+7)}\right]^{\frac{1}{2}}+i \lambda_{\Omega n} D_{m n}^{(\lambda)} .
\end{aligned}
$$

Not all $D_{m n}^{(\lambda)}$ are needed for the latter work, in fact only two are required. Solution of the recursion relation and normalization gives 


$$
\begin{aligned}
& D_{\Omega n}^{(\lambda)}=\left[\frac{(\Omega+n+4) !(\Omega-n) !(\Omega+2) !(\Omega+2) !}{\left(\frac{\lambda+\Omega-n}{2}\right) !\left(\frac{\Omega-n-\lambda}{2}\right) !\left(\frac{\Omega+n+\lambda+4}{2}\right) !\left(\frac{\Omega+n-\lambda+4}{2}\right) !(2 \Omega+4) !}\right]^{\frac{1}{2}}, \\
& D_{\Omega-1 n}^{(\lambda)}=i \lambda\left[\frac{(\Omega+n+3) !(\Omega-n-1) !(\Omega+2) !(2 \Omega+3) !}{\left(\frac{\Omega-n+\lambda}{2}\right) !\left(\frac{\Omega-n-\lambda}{2}\right) !\left(\frac{\Omega+n+\lambda+4}{2}\right) !\left(\frac{\Omega+n-\lambda+4}{2}\right) !(2 \Omega+4) !}\right]^{\frac{1}{2}} .
\end{aligned}
$$

\subsection{MATRIX ELEMENTS OF $A^{\dagger}, A$ FOR $v=0$ STATES}

The pair creation and annihilation operators $A^{\dagger}$ and $A$ are linear combinations of the operators $J_{i 7}$ and $J_{i 8}$, with $i=1,2, \ldots, 6$; (see table 1). From the point of view of the canonical chain $O(8) \supset O(7) \supset(6) \supset \ldots$ by far the simplest of these operators are the combinations $\left(J_{67} \pm i J_{68}\right)$. By using the irreducible tensor classification for the pair operators $A^{\dagger}(A)$ and applying the Wigner Eckhart theorem, it will be sufficient to calculate the matrix elements of $\left(J_{67} \pm i J_{68}\right)$ since the matrix elements of the remaining pair operators are simply related to these by known factors which are functions only of the $\mathrm{O}(6)$, spin, and isospin quantum numbers. From table 1 and eq. (2.12) it can be seen, in particular, that

$$
\begin{aligned}
-\left(J_{67}-i J_{68}\right) & =\left({ }^{13} A^{\dagger}(0,1)-{ }^{13} A^{\dagger}(0,-1)\right) \\
& =\left(T_{-1,(100), 00,11}^{(1100)}-T_{-1,(100), 00,1-1}^{(1100)} .\right.
\end{aligned}
$$

The matrix element of a component of the irreducible tensor operator $T^{(1100)}$ can be factored by the Wigner Eckart theorem. In general

$$
\begin{aligned}
& \left\langle\left(Q p p^{\prime} p^{\prime \prime}\right) \lambda_{2}\left(P_{2} P_{2}^{\prime} P_{2}^{\prime \prime}\right) S_{2} M_{S_{2}} T_{2} M_{T_{2}}\left|T_{\Delta \lambda,(100), S_{0} M s_{0}, T_{0} M_{T_{0}}}^{(1100)}\right|\right. \\
& \left.\times\left(Q p p^{\prime} p^{\prime \prime}\right) \lambda_{1}\left(P_{1} P_{1}^{\prime} P_{1}^{\prime \prime}\right) S_{1} M_{S_{1}} T_{1} M_{T_{1}}\right\rangle \\
& =F\left(\left(Q p p^{\prime} p^{\prime \prime}\right) ; \lambda_{1}, \lambda_{2} ;\left(P_{1} P_{1}^{\prime} P_{1}^{\prime \prime}\right),\left(P_{2} P_{2}^{\prime} P_{2}^{\prime \prime}\right)\right)
\end{aligned}
$$

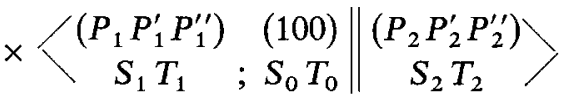

$$
\begin{aligned}
& \times\left\langle S_{1} M_{S_{1}} S_{0} M_{S_{0}} \mid S_{2} M_{S_{2}}\right\rangle\left\langle T_{1} M_{T_{1}} T_{0} M_{T_{0}} \mid T_{2} M_{T_{2}}\right\rangle .
\end{aligned}
$$

The $F$ factor which carries the sole dependence on the $O(8)$ quantum numbers and nucleon number $(\lambda)$, and depends on the $O(6)$ quantum numbers, is the product of the reduced matrix element of the operator and the reduced Wigner coefficient for the $\mathrm{O}(8) / \mathrm{O}(6)$ segment in the group decomposition. The double-barred coefficient is a reduced Wigner coefficient for the $\mathrm{O}(6) \supset[\mathrm{O}(3) \times \mathrm{O}(3)]$ segment, of the type calculated in ref. ${ }^{8}$ ). The $M_{S}$ and $M_{T}$ dependent factors are ordinary angular momentum Wigner coefficients for spin and isospin space. For the case $v=0$ both the $O(8)$ and 
$\mathrm{O}(6)$ representations are specified by a single integer; $\left(Q p p^{\prime} p^{\prime \prime}\right)=(\Omega 000),\left(P P^{\prime} P^{\prime \prime}\right)=$ $(n 00)$. It will therefore be convenient to use an abbreviated notation for the $F$ factor. In this case eq. (3.9) is written

$$
\begin{gathered}
\left\langle(\Omega 000) \lambda_{2}\left(n_{2} 00\right) S_{2} M_{S_{2}} T_{2} M_{T_{2}}\left|T_{\Delta \lambda,(100), S_{0} M_{S_{0}} T_{0} M_{T_{0}}}^{(1100)}\right|(\Omega 000) \lambda_{1}\left(n_{1} 00\right) S_{1} M_{S_{1}} T_{1} M_{T_{1}}\right\rangle \\
=F\left(\Omega ; \lambda_{1}, \lambda_{2} ; n_{1}, n_{2}\right)\langle\begin{array}{c}
{\left[n_{1} n_{1} 0\right]} \\
S_{1} T_{1} ;
\end{array} \underbrace{[110]}_{S_{0} T_{0}}|| \begin{array}{c}
\left.n_{2} n_{2} 0\right] \\
S_{2} T_{2}
\end{array}\rangle \\
\times\left\langle S_{1} M_{S_{1}} S_{0} M_{S_{0}} \mid S_{2} M_{S_{2}}\right\rangle\left\langle T_{1} M_{T_{1}} T_{0} M_{T_{0}} \mid T_{2} M_{T_{2}}\right\rangle .
\end{gathered}
$$

In the double-barred (Wigner supermultiplet) coefficients, the $\mathrm{O}(6)$ representation labels, (e.g., $\left.\left(n_{1} 00\right)\right)$, have been replaced with their SU(4) equivalent, $\left(\left[n_{1} n_{1} 0\right]\right)$, since the tabulations of ref. ${ }^{8}$ ) make use of the SU(4) notation for the Wigner supermultiplet scheme. To calculate the desired $F$ factors any simple choice of spin and isospin quantum numbers can be made. In the $O(6)$ representation ( $n 00)$ the highest weight state, $S=M_{S}=n, T=M_{T}=0$, and its "nearest neighbor", $S=M_{S}=n-1$, $T=M_{T}=1$, will be sufficient. In terms of the $\mathrm{O}(8) \supset \mathrm{O}(7) \supset \mathrm{O}(6) \supset \ldots \supset \mathrm{O}(2)$ state vectors as defined in (2.6), these two states have a very simple form. The highest weight state is given by

$$
\left|(\Omega 000) \lambda(n 00), S=M_{S}=n, T=M_{T}=0\right\rangle=\sum_{m=n}^{\Omega} D_{m n}^{(\lambda)}\left|\begin{array}{cccc}
\Omega & 0 & 0 & 0 \\
m & 0 & 0 \\
n & 0 & 0 \\
n & 0 & \\
n & 0 & \\
n & & \\
n &
\end{array}\right|
$$

The state with $S=M_{S}=n-1, T=: M_{T}=1$, can be obtained from the highest weight state by acting on it with the appropriate step-down operator, (as defined in ref. $\left.{ }^{8}\right)$ ), if the latter is expressed in terms of the $J_{\alpha \beta}$.

$\left|(\Omega 000) \lambda(n 00), S=M_{S}=n-1, T=M_{T}=1\right\rangle$

$$
=-\frac{1}{\sqrt{2}} \sum_{m=n}^{\Omega} D_{m n}^{(\lambda)}\left\{\begin{array}{cccc}
\Omega & 0 & 0 & 0 \\
m & 0 & 0 \\
n & 0 & 0 \\
n & 0 & \\
n-1 & 0 & \\
n-1 & &
\end{array}|+i| \begin{array}{cccc}
\Omega & 0 & 0 & 0 \\
m & 0 & 0 \\
n & 0 & 0 \\
n-1 & 0 & \\
n-1 & 0 & \\
n-1 & & \\
n-1 & &
\end{array}\right\} \text {. }
$$

To calculate the $F$ factor $F(\Omega ; \lambda, \lambda-1 ; n-1, n)$, for example, we use eqs. (3.8) and (3.10) with the above choices for spin and isospin quantum numbers. 


$$
\begin{aligned}
& \left\langle(\Omega 000), \lambda-1,(n 00) S_{2}=M_{S_{2}}=n-1, T_{2}=M_{T_{2}}=1\left|i\left(J_{67}-i J_{68}\right)\right|\right. \\
& \left.(\Omega 000), \lambda,(n-1,0,0) S_{1}=M_{S_{1}}=n-1, T_{1}=M_{T_{1}}=0\right\rangle \\
& =F(\Omega ; \lambda(\lambda-1) ; n-1, n)\left\langle\begin{array}{c|c}
{[n-1 n-10} & 0 \\
n-1,0
\end{array} ; \begin{array}{c}
{[110]} \\
01
\end{array} \mid \begin{array}{c}
{[n n 0]} \\
n-11
\end{array}\right\rangle \\
& \times[\langle n-1 n-100 \mid n-1 n-1\rangle\langle 0011 \mid 11\rangle \\
& -\langle n-1, n-100 \mid n-1 n-1\rangle\langle 001-1 \mid 11\rangle] \text {. }
\end{aligned}
$$

On the right hand side, all but the $F$ factor are known coefficients. (The ordinary angular momentum coupling coefficients all have the value +1 , with the exception of $\langle 001-1 \mid 11\rangle$ which is of course equal to zero.) The left hand side of eq. (3.13) can be calculated from the known matrix elements ${ }^{6}$ ) of $J_{67}$ and $J_{78}$ in the canonical basis of $\mathrm{O}(8) \supset \mathrm{O}(7) \supset \mathrm{O}(6) \supset \ldots$ The matrix element of $\left(J_{67}-i J_{68}\right)$ in this basis must be diagonal in the quantum numbers for $\mathrm{O}(5)$ and its subgroups, and is a function only of the quantum numbers for $O(8)$ through $O(5)$, inclusively. It is thus convenient to use the short hand notation

$$
\Psi_{\Omega n p}^{(\lambda)} \equiv \sum_{m=n}^{\Omega} D_{m n}^{(\lambda)} \varphi_{\Omega m n p} \equiv \sum_{m=n}^{\Omega} D_{m n}^{(\lambda)}\left|\begin{array}{cccc}
\Omega & 0 & 0 & 0 \\
m & 0 & 0 & \\
n & 0 & 0 & \\
p & 0 & & \\
\vdots & \cdot & &
\end{array}\right|
$$

where, in the last term, all the quantum numbers for the subspace of $\mathrm{O}(5)$ are omitted since, for present purposes, they can have any arbitrary value.

The matrix elements of $J_{78}$ and $J_{67}$ in the $\varphi_{\Omega m n p}$ scheme are known

$$
\begin{aligned}
& J_{78} \varphi_{\Omega m n p}=A_{\Omega m n+} \varphi_{\Omega m+1 n p}+A_{\Omega m n-} \varphi_{\Omega m-1 n p}, \\
& J_{67} \varphi_{\Omega m n p}=B_{m n p+} \varphi_{\Omega m n+1 p}+B_{m n p}-\varphi_{\Omega m n-1 p},
\end{aligned}
$$

where the coefficients $A$ have been given in eq. (3.4), and

$$
\begin{gathered}
B_{m n p+}=-\frac{i}{2}\left[\frac{(n-p+1)(n+p+4)(m-n)(m+n+5)}{(n+2)(n+3)}\right]^{\frac{1}{2}}, \\
B_{m n p-}=\frac{i}{2}\left[\frac{(n-p)(n+p+3)(m-n+1)(m+n+4)}{(n+1)(n+2)}\right]^{\frac{1}{2}} .
\end{gathered}
$$

To calculate $J_{67} \pm i J_{68}$ we use the commutation relations (2.5),

which, with

$$
J_{67} \pm i J_{68}=J_{67} \pm\left(J_{78} J_{67}-J_{67} J_{78}\right)
$$

$$
J_{78} \Psi_{\Omega n p}^{(\lambda)}=\lambda \Psi_{\Omega n p}^{(\lambda)}
$$


gives

$$
\begin{aligned}
& \left(J_{67}+i J_{68}\right) \Psi_{\Omega n p}^{(\lambda)}=c_{\lambda \Omega n p}^{+} \Psi_{\Omega(n+1) p}^{(\lambda+1)}+c_{\lambda \Omega n p}^{-} \Psi_{\Omega(n-1) p}^{(\lambda+1)} \\
& \left(J_{67}-i J_{68}\right) \Psi_{\Omega n p}^{(\lambda)}=b_{\lambda \Omega n p}^{+} \Psi_{\Omega(n+1) p}^{(\lambda-1)}+b_{\lambda \Omega n p}^{-} \Psi_{\Omega(n-1) p}^{(\lambda-1)}
\end{aligned}
$$

with

$$
\begin{gathered}
c_{i \Omega n p}^{+}=-\frac{i}{2}\left[\frac{(n-p+1)(n+p+4)(\Omega+n+\lambda+6)(\Omega-n-\lambda)}{(n+2)(n+3)}\right]^{\frac{1}{2}}, \\
c_{i \Omega n p}^{-}=\frac{i}{2}\left[\frac{(n-p)(n+p+3)(\Omega-n+\lambda+2)(\Omega+n-\lambda+4)}{(n+2)(n+1)}\right]^{\frac{1}{2}},
\end{gathered}
$$

and

$$
\begin{aligned}
& b_{\lambda \Omega n p}^{+}=-c_{(\lambda-1) \Omega(n+1) p}^{-}, \\
& b_{\lambda \Omega n p}^{-}=-c_{(\lambda-1) \Omega(n-1) p}^{+} .
\end{aligned}
$$

With eqs. (3.11), (3.12), (3.14), (3.20), and (3.22), it can be seen that the left hand side of eq. (3.13) has the value

$$
\frac{i(-i)}{\sqrt{ } 2} b_{\lambda \Omega(n-1) p=(n-1)}^{+}=-\frac{1}{\sqrt{ } 2} c_{(\lambda-1) \Omega n p=(n-1)}^{-} .
$$

Using the tabulated value ${ }^{8}$ ) for the SU(4) Wigner coefficient of the right hand side of eq. (3.13), we obtain

$$
\begin{aligned}
F(\Omega ; \lambda, \lambda-1 ; n-1, n) & =-\left[\frac{1}{2} n\right]^{\frac{1}{2}} c_{(\lambda-1) \Omega n p=(n-1)}^{-} \\
& =\frac{1}{2}\left[\frac{n(\Omega-n+\lambda+1)(\Omega+n-\lambda+5)}{(n+2)}\right]^{\frac{1}{2}} .
\end{aligned}
$$

The other $F$ factors are obtained by similar techniques. The results are tabulated in table 3 .

TABLE 3

$F$ factors for $v=0$ states

$$
\begin{aligned}
& F(\Omega ; \lambda-1, \lambda ; n-1, n)=\frac{1}{2}\left[\frac{n(\Omega+n+\lambda+4)(\Omega-n-\lambda+2)}{(n+2)}\right]^{\frac{1}{2}} \\
& F(\Omega ; \lambda-1, \lambda ; n+1, n)=\frac{1}{2}\left[\frac{(n+4)(\Omega+n-\lambda+6)(\Omega-n+\lambda)}{(n+2)}\right]^{\frac{1}{2}} \\
& F(\Omega ; \lambda+1, \lambda ; n-1, n)=\frac{1}{2}\left[\frac{n(\Omega-n+\lambda+2)(\Omega+n-\lambda+4)}{(n+2)}\right]^{\frac{1}{2}} \\
& F(\Omega ; \lambda+1, \lambda ; n+1, n)=\frac{1}{2}\left[\frac{(n+4)(\Omega+n+\lambda+6)(\Omega-n-\lambda)}{(n+2)}\right]^{\frac{1}{2}}
\end{aligned}
$$




\subsection{THE $F$ FACTORS FOR $v=1$ STATES}

The technique used to calculate the $F$ factors for the $v=1$ states is similar to that for the $v=0$ states. The main difference comes from the fact that the $\mathrm{O}(8)$ irreducible representation which, for $v=1$ is characterized by $\left(\Omega-\frac{1}{2}, \frac{1}{2} \frac{1}{2} \frac{1}{2}\right)$ contains two possible types of $\mathrm{O}(6)$ representations, namely $\left(n+\frac{1}{2}, \frac{1}{2} \frac{1}{2}\right)$ or $\left(n+\frac{1}{2}, \frac{1}{2},-\frac{1}{2}\right)$ corresponding to the $\mathrm{SU}(4)$ representation $[n+1, n, 0]$ and $[n+1, n+1,1]$, which are conjugate to each other. The corresponding $\mathrm{U}(4)$ representations which give the total number of nucleons are $[n+1+i, n+i, i, i]$ and $[n+1+i, n+1+i, i+1, i]$, respectively; where $i=0,1,2, \ldots(\Omega-n-1)$. The nucleon numbers are therefore given by: $N=2 n+$ $+1+4 i$ for $\left(n+\frac{1}{2}, \frac{1}{2} \frac{1}{2}\right)$, and $N=2 n+3+4 i$ for $\left(n+\frac{1}{2}, \frac{1}{2}-\frac{1}{2}\right)$. The corresponding eigenvalues, $\lambda$, of the operator $Q_{0}=J_{78}=\left(\Omega-\frac{1}{2} N_{\mathrm{op}}\right)$ are

$$
\begin{aligned}
& \text { for }\left(n+\frac{1}{2}, \frac{1}{2}, \frac{1}{2}\right) ; \lambda=\Omega-n-2 i-\frac{1}{2}, \\
& \text { for }\left(n+\frac{1}{2}, \frac{1}{2},-\frac{1}{2}\right) ; \lambda=-\Omega+n+2 i+\frac{1}{2}, i=0,1,2, \ldots,(\Omega-n-1) \text {. }
\end{aligned}
$$

\section{TABLE 4}

$F$ factors for $v=1$ states

$$
\begin{array}{ll}
F(\Omega ; \lambda-1, \lambda ; n-1, n) & =\frac{1}{4}\left[\frac{n(2 \Omega-2 n-2 \lambda+3)(2 \Omega+2 n+2 \lambda+9)}{(n+2)}\right]^{\frac{1}{2}} \\
F\left(\Omega ; \lambda-1, \lambda ; n^{*}, n\right) & =\frac{i}{2}\left[\frac{(2 \Omega+2 n+2 \lambda+9)(2 \Omega-2 n-2 \lambda+11)}{2(n+2)(n+3)}\right]^{\frac{1}{2}} \\
F(\Omega ; \lambda-1, \lambda ; n+1, n) & =\frac{1}{4}\left[\frac{(n+5)(2 \Omega+2 n-2 \lambda+11)(2 \Omega-2 n+2 \lambda-3)}{(n+3)}\right]^{\frac{1}{2}} \\
F(\Omega ; \lambda+1, \lambda ; n-1, n) & =\frac{1}{4}\left[\frac{n(2 \Omega+2 n-2 \lambda+7)(2 \Omega-2 n+2 \lambda+1)}{(n+2)}\right]^{\frac{1}{2}} \\
F\left(\Omega ; \lambda+1, \lambda ; n^{*}, n\right) & =\frac{i}{2}\left[\frac{(2 \Omega-2 n+2 \lambda+1)(2 \Omega-2 n-2 \lambda-1)}{2(n+2)(n+3)}\right]^{\frac{1}{2}} \\
F(\Omega ; \lambda+1, \lambda ; n+1, n) & =\frac{1}{4}\left[\frac{(n+5)(2 \Omega-2 n-2 \lambda-1)(2 \Omega+2 n+2 \lambda+13)}{(n+3)}\right]^{\frac{1}{2}} \\
F\left(\Omega ; \lambda-1, \lambda ;(n-1)^{*}, n^{*}\right) & =\frac{1}{4}\left[\frac{n(2 \Omega-2 n-2 \lambda+1)(2 \Omega+2 n+2 \lambda+7)}{(n+2)}\right]^{\frac{1}{2}} \\
F\left(\Omega ; \lambda-1, \lambda ; n, n^{*}\right) & =\frac{-i}{2}\left[\frac{(2 \Omega-2 n+2 \lambda-1)(2 \Omega-2 n-2 \lambda+1)}{2(n+2)(n+3)}\right]^{\frac{1}{2}} \\
F\left(\Omega ; \lambda-1, \lambda ;(n+1)^{*}, n^{*}\right) & =\frac{1}{4}\left[\frac{(n+5)(2 \Omega+2 n-2 \lambda+13)(2 \Omega-2 n+2 \lambda-1)}{(n+3)}\right]^{\frac{1}{2}} \\
F\left(\Omega ; \lambda+1, \lambda ;(n-1)^{*}, n^{*}\right) & =\frac{1}{4}\left[\frac{n(2 \Omega-2 n+2 \lambda+3)(2 \Omega+2 n-2 \lambda+9)}{(n+2)}\right]^{\frac{1}{2}} \\
F\left(\Omega ; \lambda+1, \lambda ; n, n^{*}\right) & =\frac{-i}{2}\left[\frac{(2 \Omega+2 n+2 \lambda+11)(2 \Omega-2 n-2 \lambda+9)}{2(n+2)(n+3)}\right]^{\frac{1}{2}} \\
F\left(\Omega ; \lambda+1, \lambda ;(n+1)^{*}, n^{*}\right) & =\frac{1}{4}\left[\frac{(n+5)(2 \Omega-2 n-2 \lambda-3)(2 \Omega+2 n+2 \lambda+11)}{(n+3)}\right]^{\frac{1}{2}} \\
&
\end{array}
$$


that is, the two sets of eigenvalues for the conjugate $O(6)$ representation in $\left(\Omega-\frac{1}{2}, \frac{11}{2} \frac{1}{2}\right)$ are of opposite sign but equal in magnitude. The expansion coefficients, $D$, analogous to those of eq. (3.2), therefore, have the property: the coefficient $D$ for $\left(n+\frac{1}{2}, \frac{1}{2},-\frac{1}{2}\right)$ and $-\lambda$ is the complex conjugate of the coefficient $D$ for $\left(n+\frac{1}{2}, \frac{1}{2} \frac{1}{2}\right)$ and $+\lambda$. Details are given in ref. ${ }^{9}$ ). Techniques similar to those of subsect. 3.2 give the $F$ factors for the $v=1$ states. The results are shown in table 4 . In the argument for the $F$ factors $\Omega$ is now short hand notation for the $\mathrm{O}(8)$ label $\left(\Omega-\frac{1}{2}, \frac{1}{2} \frac{11}{2}\right), n$ stands for the $\mathrm{O}(6)$ representation $\left(n+\frac{1}{2}, \frac{1}{2} \frac{1}{2}\right)$ or $\mathrm{SU}(4)$ representation $[n+1, n, 0]$; while $n^{*}$ stands for the conjugate representation $\left(n+\frac{1}{2}, \frac{1}{2},-\frac{1}{2}\right)$ or its $\operatorname{SU}(4)$ equivalent $[n+1, n+1,1]$. With the $F$ factors of table 4 , and the table of SU(4) Wigner coefficients of ref. ${ }^{8}$ ), the matrix elements of $A^{+}$can again be obtained by the use of eq. (2.12) and the Wigner Eckart theorem, eq. (3.9).

\section{Some applications of the quasispin method}

With the matrix elements of the pair creation and annihilation operators calculated in the last section, exact solutions can be found for the pairing problem involving states of seniority $v=0$ or 1 .

\subsection{THE PAIRING HAMILTONIAN}

The general nuclear Hamiltonian is assumed to have the form

$$
H=H_{\text {s.p. }}+H_{2-\text { body }}
$$

where the single-particle Hamiltonian is given by

$$
H_{\text {s.p. }}=\sum_{i} \varepsilon_{i} N_{i}, \quad \text { with } N_{i}=\sum_{m_{i} m_{s} m_{t}} a_{i m_{i} m_{s} m_{t}}^{+} a_{i m_{i} m_{s} m_{t}} .
$$

The indices $i$ characterize the single-particle levels, and $\varepsilon_{i}$ are the single-particle energies. The spatial degeneracy of the levels is given by $\Omega_{i}=\sum_{m_{i}} 1$, for example $\Omega_{i}=2 l_{i}+1$ if $i$ denotes $l_{i}$. In pairing model studies the general two-body interaction is replaced by a pairing Hamiltonian. In the $\Omega S T$ scheme the pairing Hamiltonian acting on states of a nuclear configuration consisting of several single particle energy levels can be written in the most general form as

$$
\begin{aligned}
H_{\text {pairing }} & =\sum_{i, j} g_{31}^{i j} \sum_{M_{S}}{ }^{31} A_{i}^{+}\left(M_{S}, 0\right)^{31} A_{j}\left(M_{S}, 0\right) \\
& +\sum_{i, j} g_{13}^{i j} \sum_{M_{T}}{ }^{13} A_{i}^{+}\left(0, M_{T}\right)^{13} A_{j}\left(0, M_{T}\right) .
\end{aligned}
$$

The $g_{(2 S+1)(2 T+1)}^{i j}$ give the strength of the pairing interaction between a pair of particles coupled to $L=0, S T$, in the $i$ th single-particle level and a similar pair of particles in the $j$ th single-particle level. Since this Hamiltonian is a scalar in both spin and isospin space, its eigenvalues are independent of $M_{\mathrm{S}}$ and $M_{T}$, and the total 
$S$ and $T$ of the system are good quantum numbers. In general, however, the Hamiltonian (4.3) can be off-diagonal in the Wigner supermultiplet quantum numbers. If a further degeneration is made in the pairing Hamiltonian by setting $g_{31}^{i j}=g_{13}^{i i}$, the Hamiltonian becomes an $\mathrm{O}(6)$, (or SU (4)), scalar as well. In this case the total Wigner supermultiplet is a good quantum number, and the eigenvalues of (4.3) are independent of $S$ and $T$. The different $S, T$ states of a given Wigner supermultiplet become degenerate. Since the $A_{i}^{+}$and $A_{j}$ are infinitesimal operators for $\mathrm{O}(8)$, the Hamiltonian is diagonal in the seniority quantum numbers for the various singleparticle levels $i$; that is, $v_{i} p_{i} p_{i}^{\prime} p_{i}^{\prime \prime}$ are good quantum numbers for all $i$. Finally, if both the parameters $g^{i j}$ and $\varepsilon_{i}$ are independent of $i$ and $j$, the overall $\mathrm{O}(8)$ quantum numbers $\left(\Omega-\frac{1}{2} v, p p^{\prime} p^{\prime \prime}\right)$ become good quantum numbers, where the $\left(\Omega-\frac{1}{2} v, p p^{\prime} p^{\prime \prime}\right)$ describe the $O(8)$ representations obtained from the direct products of the $O(8)$ representations $\left(\Omega_{i}-\frac{1}{2} v_{i}, p_{i} p_{i} p_{i}^{\prime} p_{i}^{\prime \prime}\right)$ for the various single-particle levels.

Since our interest is in the most general case, the base vectors in this investigation are to be designated by the seniority, Wigner supermultiplet, spin, and isospin quantum numbers for the individual single-particle levels $i$. Applications will be illustrated in detail for systems with even nucleon number and individual single-particle level seniorities $v_{i}=0$. For such a state

$$
\begin{aligned}
A_{i}^{+}\left(M_{S_{0}}, M_{T_{0}}\right)\left|\Omega_{i} \lambda_{i}\left[n_{i}^{\prime} n_{i}^{\prime} 0\right] S_{i}^{\prime} M_{S}^{\prime}, T_{i}^{\prime} M_{T_{0}}^{\prime}\right\rangle \\
=\sum_{\substack{n_{i} S_{i} M_{S_{i}} \\
T_{i} M T_{i}}}\left|\Omega_{i}\left(\lambda_{i}-1\right)\left[n_{i} n_{i} 0\right] S_{i} M_{S_{i}}, T_{i} M_{T_{i}}\right\rangle A_{\Omega_{i} \lambda_{i} i_{i}^{\prime} S_{i}^{\prime} M^{\prime} S_{i} T^{\prime} i M^{\prime} T_{i}}^{\Omega_{i}\left(\lambda_{i}-1\right) n_{i} S_{i} M S_{S_{i}} T_{i} M T_{i}},
\end{aligned}
$$

with $n_{i}=n_{i}^{\prime} \pm 1$. From the results of sect. 3

$$
\begin{aligned}
A_{\Omega \lambda n^{\prime} S^{\prime} M^{\prime} S^{\prime} T^{\prime} M^{\prime} T}^{\Omega(\lambda-1) S M_{T}}=F(\Omega ; \lambda, \lambda-1 ; & \left.n^{\prime}, n\right)\left\langle\begin{array}{c}
{\left[n^{\prime} n^{\prime} 0\right]} \\
S^{\prime} T^{\prime} ;
\end{array} ; \begin{array}{c}
{[110]} \\
S_{0} T_{0}
\end{array} \| \begin{array}{c}
{[n n 0]} \\
S T
\end{array}\right\rangle \\
& \times\left\langle S^{\prime} M_{S}^{\prime} S_{0} M_{S_{0}} \mid S M_{S}\right\rangle\left\langle T^{\prime} M_{T}^{\prime} T_{0} M_{T_{0}} \mid T M_{T}\right\rangle,
\end{aligned}
$$

where level subscripts $i$ have been omitted. The needed $F$ factors are given in table 3, and the $\mathrm{SU}(4)$ Wigner coefficients in the tables of the appendix of ref. ${ }^{8}$ ). Matrix elements of the pair annihilation operators $A$ can be obtained from the matrix elements of $A^{+}$by hermitian conjugation.

\subsection{THE SINGLE LEVEL CASE, $v=0$}

As the simplest example, the problem of a pairing interaction with different spin singlet and triplet strengths acting on a system with a single well isolated single-particle level will be treated first. The matrix element of the pairing Hamiltonian is diagonal in all but the $\mathrm{O}(6)$ quantum number, and for $v=0$ has the form 


$$
\begin{aligned}
& H_{\bar{n}, n}=\left\langle\begin{array}{c}
\Omega \lambda \bar{n} \\
\left(S M_{S}\right)\left(T M_{T}\right)
\end{array}\left|H_{\text {pairing }}\right| \begin{array}{c}
\Omega \lambda n \\
\left(S M_{S}\right)\left(T M_{T}\right)
\end{array}\right\rangle \\
& =g_{31} \sum_{S^{\prime \prime} M_{S^{\prime \prime}} n^{\prime \prime}} A_{\Omega \lambda+1 n^{\prime \prime} S^{\prime \prime} M_{S^{\prime \prime}} T M_{T}}^{\Omega \lambda \bar{n} S M_{T} T M_{T}} A_{\Omega \lambda+1 n^{\prime \prime} S^{\prime \prime} M_{S^{\prime \prime}} T M_{T}}^{\Omega \lambda n S M_{S} T M_{T}} \\
& +g_{13} \sum_{T^{\prime \prime} M_{T^{\prime \prime}} n^{\prime \prime}} A_{\Omega \lambda+1 n^{\prime \prime} S M_{S} T^{\prime \prime} M^{\prime \prime} T}^{\Omega \lambda \bar{n} S M M_{T} T \lambda+1 n^{\prime \prime} S M_{S} T^{\prime \prime} M_{T^{\prime \prime}}} A_{\Omega \lambda}^{\Omega \lambda n S M_{S} T M^{\prime}}
\end{aligned}
$$

Since $A^{+}$(or $A$ ) connect $n$ to $n \pm 1$ only, $\bar{n}$ can have the values $n, n+2$, and $n-2$; and there are three types of matrix elements.

(a) Pairing Hamiltonian connection $n \rightarrow \bar{n}=n$.

$$
\begin{aligned}
H_{n n}= & g_{13}\left\{\frac{(\Omega+n+\lambda+6)(\Omega-n-\lambda)}{8(n+2)(n+3)}\right. \\
& \times\left[\frac{(S+1)(n+S+T+4)(n+S-T+3)+S(n-S+T+3)(n-S-T+2)}{2 S+1}\right] \\
& +\frac{(\Omega-n+\lambda+2)(\Omega+n-\lambda+4)}{8(n+1)(n+2)} \\
& \left.\times\left[\frac{(S+1)(n-S+T+1)(n-S-T)+S(n+S-T+1)(n+S+T+2)}{2 S+1}\right]\right\} \\
& \left.\left.+g_{31} \frac{\left(\frac{(\Omega+n+\lambda+6)(\Omega-n-\lambda)}{8(n+2)(n+3)}\right.}{2 T+1}\right]\right\} \\
& \times\left[\frac{(T+1)(n+S+T+4)(n-S+T+3)+T(n-T+S+3)(n-S-T+2)}{2 T+1}\right] \\
& +\frac{(\Omega-n+\lambda+2)(\Omega+n-\lambda+4)}{8(n+2)(n+1)} \\
& \times\left[\frac{(T+1)(n-T+S+1)(n-S-T)+T(n+T-S+1)(n+S+T+2)}{2}\right.
\end{aligned}
$$

(b) Pairing Hamiltonian connection $n \rightarrow \bar{n}=n \pm 2$

$$
\begin{aligned}
H_{n+2, n}=\left(g_{31}-g_{13}\right) & \frac{1}{8(n+3)}\left[\frac{(\Omega+n+\lambda+6)(\Omega+n-\lambda+6)(\Omega-n+\lambda)(\Omega-n-\lambda)}{(n+2)(n+3)}\right. \\
& \times(n+S+T+4)(n+S-T+3)(n-S+T+3)(n-S-T+2)]^{\frac{1}{2}},
\end{aligned}
$$

and $H_{n-2, n}=H_{n, n-2}$.

The size of the matrix depends on the nucleon number $N$, (or $\lambda=\Omega-\frac{1}{2} N$ ), and the specific values of $S$ and $T$. The possible Wigner supermultiplets and their $S, T$ values 
for some of the smaller nucleon numbers are shown in table 5 . For $N=4$, for example, it can be seen that the Hamiltonian matrix is $1 \times 1$ for the $(S T)$ values $(20),(11)$, and $(02)$, but is $2 \times 2$ for $(S T)=(00)$. For $N=4,(S T)=(00)$, the Hamiltonian matrix (4.7) has the specific form

$$
H_{\text {pairing }}=\left(g_{13}+g_{31}\right)^{2}\left(\begin{array}{cc}
\Omega+2 & \Delta\left[\frac{1}{3}(\Omega+2)(\Omega-1)\right]^{\frac{1}{2}} \\
\Delta\left[\frac{1}{3}(\Omega+2)(\Omega-1)\right]^{\frac{1}{2}} & \Omega-1
\end{array}\right),
$$

where

$$
\Delta=\frac{2\left(g_{31}-g_{13}\right)}{g_{31}+g_{13}}
$$

For $N=4$ and $v=0$, the pairing energies therefore have the values

$$
\begin{aligned}
& E_{(00)}^{N=4}=\frac{1}{2}\left(g_{13}+g_{31}\right)\left\{(2 \Omega+1) \pm\left[(2 \Omega+1)^{2}-4(\Omega+2)(\Omega-1)\left(1-\frac{1}{3} \Delta^{2}\right)\right]^{\frac{1}{2}}\right\} \\
& E_{(20)}^{N=4}=\left(g_{13}+g_{31}\right)(\Omega-1)(1-\Delta) \\
& E_{(02)}^{N=4}=\left(g_{13}+g_{31}\right)(\Omega-1)(1+\Delta) \\
& E_{(11)}^{N=4}=\left(g_{13}+g_{31}\right)(\Omega-1) .
\end{aligned}
$$

\begin{tabular}{|c|c|c|c|c|}
\hline$N$ & $\lambda$ & U(4) & $\begin{array}{l}\mathrm{SU} \\
n\end{array}$ & $(S, T)$ \\
\hline 2 & $\Omega-1$ & [11] & 1 & $(10)(01)$ \\
\hline \multirow{2}{*}{4} & \multirow{2}{*}{$\Omega-2$} & [1111] & 0 & $(00)$ \\
\hline & & [22] & 2 & $\begin{array}{r}(20)(11)(02) \\
(00)\end{array}$ \\
\hline \multirow{2}{*}{6} & \multirow{2}{*}{$\Omega-3$} & [2211] & 1 & $(10)(01)$ \\
\hline & & [33] & 3 & $\begin{array}{r}(30)(21)(12)(03) \\
(10)(01)\end{array}$ \\
\hline \multirow{3}{*}{8} & \multirow{3}{*}{$\Omega-4$} & [2222] & 0 & $(00)$ \\
\hline & & [3311] & 2 & $\begin{array}{r}(20)(11)(02) \\
(00)\end{array}$ \\
\hline & & [44] & 4 & $\begin{array}{r}(40)(31)(22)(13)(04) \\
(20)(11)(02) \\
(00)\end{array}$ \\
\hline
\end{tabular}

For general nucleon number $N, v=0$, the pairing Hamiltonian will give rise to a $k \times k$ matrix for $(S T)$ values such that $S+T=\frac{1}{2} N-2(k-1)$.

TABLE 5

Wigner supermultiplet classification of the $v=0$ states for particle numbers $N=2,4,6$ and 8 
When $g_{13}=g_{31}$ the pairing Hamiltonian has higher symmetry. The Wigner supermultiplet number becomes a good quantum number. The Hamiltonian matrix (4.7) becomes diagonal in $n$, and the matrix elements are independent of $S$ and $T$, so that the various states $(S T)$ of a given supermultiplet become degenerate. In the case $g_{13}=g_{31}=g$, eq. (4.7) contracts to

$$
E_{\Omega n \lambda}=\frac{1}{2} g\left(\Omega^{2}+6 \Omega-n^{2}-4 n-\lambda^{2}-6 \lambda\right) .
$$

For example, the five energy levels of eq. (4.9) for $N=4$ collapse into two as $\Delta \rightarrow 0$, namely

$$
\begin{aligned}
& E_{n=2}^{N=4}=2 g(\Omega-1) \quad \text { with }(S T)=(20),(11),(02),(00), \\
& E_{n=0}^{N=4}=2 g(\Omega+1) \quad \text { with }(S T)=(00) .
\end{aligned}
$$

The result (4.10) can also be expressed in terms of the Casimir invariants of $O(8)$ and $O(6)$

$$
E_{\Omega n \lambda}=\frac{1}{2} g\left(C(8)-C(6)-\lambda^{2}-6 \lambda\right)
$$

which agrees with the result derived by Flowers and Szpikowski ${ }^{3}$ ).

\subsection{FORMULATION OF THE MANY LEVEI, PROBLEM}

To study the competition between the pairing interaction and the single-particle excitations, the case of a system with several single-particle levels connected by a pairing interaction must be studied. The case of a system with two single-particle levels will be studied in detail. Both the effects of the pairing interaction within individual levels and between different levels must be taken into consideration.

The two-level case, seniority zero. There are many ways of coupling the two wave functions for $N_{1}$ nucleons of seniority $v_{1}=0$ in level 1 and $N_{2}$ nucleons of seniority $v_{2}=0$ in level 2 . One could couple the two $\mathrm{O}(8)$ representations to a resultant $\mathrm{O}(8)$ representation specified by overall seniority and reduced supermultiplet quantum numbers. This might be useful in the extreme strong pairing limit. Without coupling $O(8)$, one could also couple the two $O(6)$ representations so that the state vectors are specified by overall Wigner supermultiplet quantum numbers besides those for levels 1 and 2. This would be a useful coupling scheme if the Hamiltonian has enough symmetry to make the total supermultiplet good quantum numbers; but in this scheme the matrix elements would lead to expressions involving SU(4) Racah coefficients which have not yet been calculated. Since the pairing Hamiltonian is diagonal in the total $S$ and $T$, the most efficient coupling scheme is one in which $S_{1}$ and $S_{2}$ are coupled to total $S$, and $T_{1}$ and $T_{2}$ to total $T$. By such a coupling the size of the Hamiltonian matrix is greatly reduced. Also, the SU(2) Racah coefficients which appear in the general expressions for the matrix elements in this coupling scheme are so well known that no additional mathematical complications are introduced as would be the case in the coupling of the higher group representations. Of course, the simplest 
formulation of the matrix elements could be made in the completely uncoupled scheme; but this would lead to unnecessarily large matrices.

The coupling scheme chosen for the present work is therefore the one in which spins and isospins for the two levels are coupled, and the wave function is written as

$$
\left|\begin{array}{cc}
\Omega_{1} & \Omega_{2} \\
\lambda_{1} & \lambda_{2} \\
n_{1} & n_{2} \\
\left(S_{1} T_{1}\right) & \left(S_{2} T_{2}\right) \\
S T M_{S} M_{T}
\end{array}\right| .
$$

The pairing Hamiltonian (4.3) can be separated into four parts

$$
H=\sum_{i, j} H^{i j}=H^{11}+H^{22}+H^{21}+H^{12} .
$$

The matrix elements for $H^{11}$ and $H^{22}$ have already been calculated in subsect. 4.2. The matrix elements for $H^{11}$, for example, in the coupled scheme, eq. (4.13), are independent of $\Omega_{2} \lambda_{2} n_{2} S_{2} T_{2}$ and the total $S M_{S} T M_{T}$ and are given by eq. (4.7) if the quantum numbers $(\Omega \lambda n S T)$ in this equation are replaced by $\left(\Omega_{1} \lambda_{1} n_{1} S_{1} T_{1}\right)$. The matrix elements of $H^{21}$ and $H^{12}$ follow from the matrix elements of $A_{i}^{+}, A_{j}$, and standard angular momentum recoupling techniques. For example

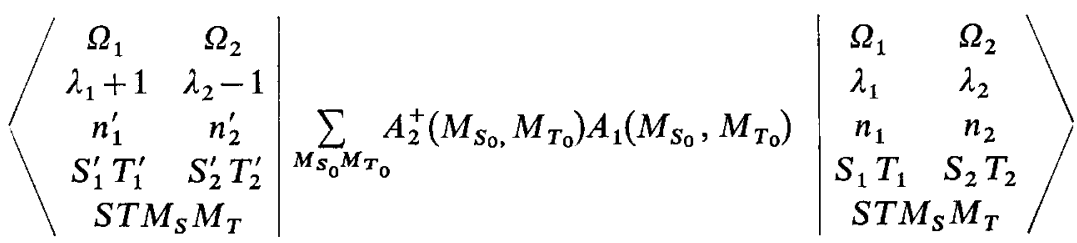

$$
\begin{aligned}
& =(-)^{S_{1}+T^{\prime}{ }_{1}+S_{2}^{\prime}+T^{\prime}{ }_{2}+S+T}\left[\left(2 S_{1}+1\right)\left(2 S_{2}^{\prime}+1\right)\left(2 T_{1}+1\right)\left(2 T_{2}^{\prime}+1\right)\right]^{\frac{1}{t}} \\
& \times\left\{\begin{array}{lll}
S_{2} & S_{2}^{\prime} & S_{0} \\
S_{1}^{\prime} & S_{1} & S
\end{array}\right\}\left\{\begin{array}{lll}
T_{2} & T_{2}^{\prime} & T_{0} \\
T_{1}^{\prime} & T_{1} & T
\end{array}\right\} \times F\left(\Omega_{1} ; \lambda_{1}+1, \lambda_{1} ; n_{1}^{\prime} n_{1}\right)
\end{aligned}
$$

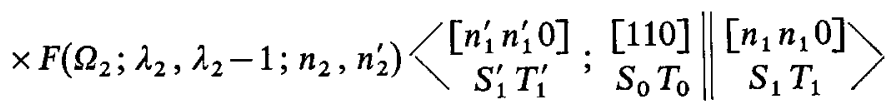

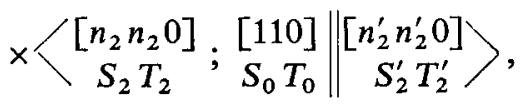

where the quantities in curly brackets are standard $6-j$ symbols in spin and isospin space.

If the wave functions for either level 1 or level 2 have $v=1$, the expression for the matrix element has the same form if the appropriate $F$ factor and double-barred $\mathrm{SU}(4)$ coefficients are replaced by the analogous factors valid for the case $v=1$.

For the many level case the most useful coupling scheme is again the one where 
the $O(8)$ and $O(6)$ representations for the various levels are left uncoupled, so that the basis is specified by the seniority and Wigner supermultiplet quantum numbers for each level $i$, as well as the spin and isospin quantum numbers $S_{i} T_{i}, i=1,2,3, \ldots$; but it will again be useful to couple the $S_{i}$ and $T_{i}$ to total resultant $S M_{S}$ and $T M_{T}$. Matrix elements of $H^{i j}$ with $i \neq j$ can now be related to those of eq. (4.15) by standard angular momentum recoupling techniques. For a three-level problem, for example, employing a basis $\left|\Omega_{1} \lambda_{1} n_{1}, \Omega_{2} \lambda_{2} n_{2}, \Omega_{3} \lambda_{3} n_{3} ;\left[\left(S_{1} T_{1} S_{2} T_{2}\right) S_{12} T_{12}, S_{3} T_{3}\right] S M_{S} T M_{T}\right\rangle$ the matrix element of $H^{21}$ follows directly from (4.15); but the matrix element of $H^{32}$, for example, would require a recoupling to an | $\left.\left[S_{1} T_{1},\left(S_{2} T_{2} S_{3} T_{3}\right) S_{23} T_{23}\right] S M_{S} T M_{T}\right\rangle$ basis with the same $\mathrm{O}(8), \lambda$, and $\mathrm{O}(6)$ quantum numbers as the above.

\subsection{COMPUTATIONS AND RESULTS}

As a specific example, a detailed computation has been carried out for a two-level problem to make it possible to study the effect of the pairing interaction in the presence of single-particle excitations. The specific example chosen is one with $\Omega_{1}=1$ and $\Omega_{2}=5$; that is one with an $l=0$ level and a nearby $l=2$ level, such as those found in the $2 \mathrm{~s}-1 \mathrm{~d}$ shell of real nuclei. For simplicity all pairing strengths are put equal to $g$. The energy reference point is chosen as the single-particle level 1 , so that $\varepsilon_{1}$ can be put equal to zero. The full Hamiltonian can then be written as

$$
H=\varepsilon N_{2}-2 g \sum_{i, j=1}^{2}\left[\sum_{M_{S}}^{31} A_{i}^{+}\left(M_{S} 0\right)^{31} A_{j}\left(M_{S} 0\right)+\sum_{M_{T}}{ }^{13} A_{i}^{+}\left(0 M_{T}\right)^{13} A_{j}\left(0 M_{T}\right)\right] .
$$

The energies for a fixed total number of particles $N=N_{1}+N_{2}$, and fixed total $S$ and $T$, can then be found by diagonalizing the matrix for the above Hamiltonian in a basis which, for seniorities $v_{1}=v_{2}=0$, can be characterized by the seven numbers $N_{1} n_{1} S_{1} T_{1} n_{2} S_{2} T_{2}$. ( $N_{2}$ is not listed simply because $\left.N_{2}=N-N_{1}\right)$. For example, for $N=4, S=T=0$ the five states of the basis are

$\begin{array}{ccccccc}N_{1} & n_{1} & S_{1} & T_{1} & n_{2} & S_{2} & T_{2} \\ 4 & 0 & 0 & 0 & 0 & 0 & 0 \\ 2 & 1 & 1 & 0 & 1 & 1 & 0 \\ 2 & 1 & 0 & 1 & 1 & 0 & 1 \\ 0 & 0 & 0 & 0 & 2 & 0 & 0 \\ 0 & 0 & 0 & 0 & 0 & 0 & 0\end{array}$

The Hamiltonian for each $N, S, T$ is then diagonalized to give the energies and wave functions for specific values of the parameter $\frac{z}{g}$. Since the total Wigner-supermultiplet degeneracy is not removed by the Hamiltonian (4.16), with one common value for $g$, states with different $S T$ values belonging to the same total Wigner supermultiplet will have to be degenerate. This can serve as a check on the numerical calculations which have been carried out for all $S T$.

Calculations have been carried out in particular for $N=4,6,8$, and 10 . The re- 


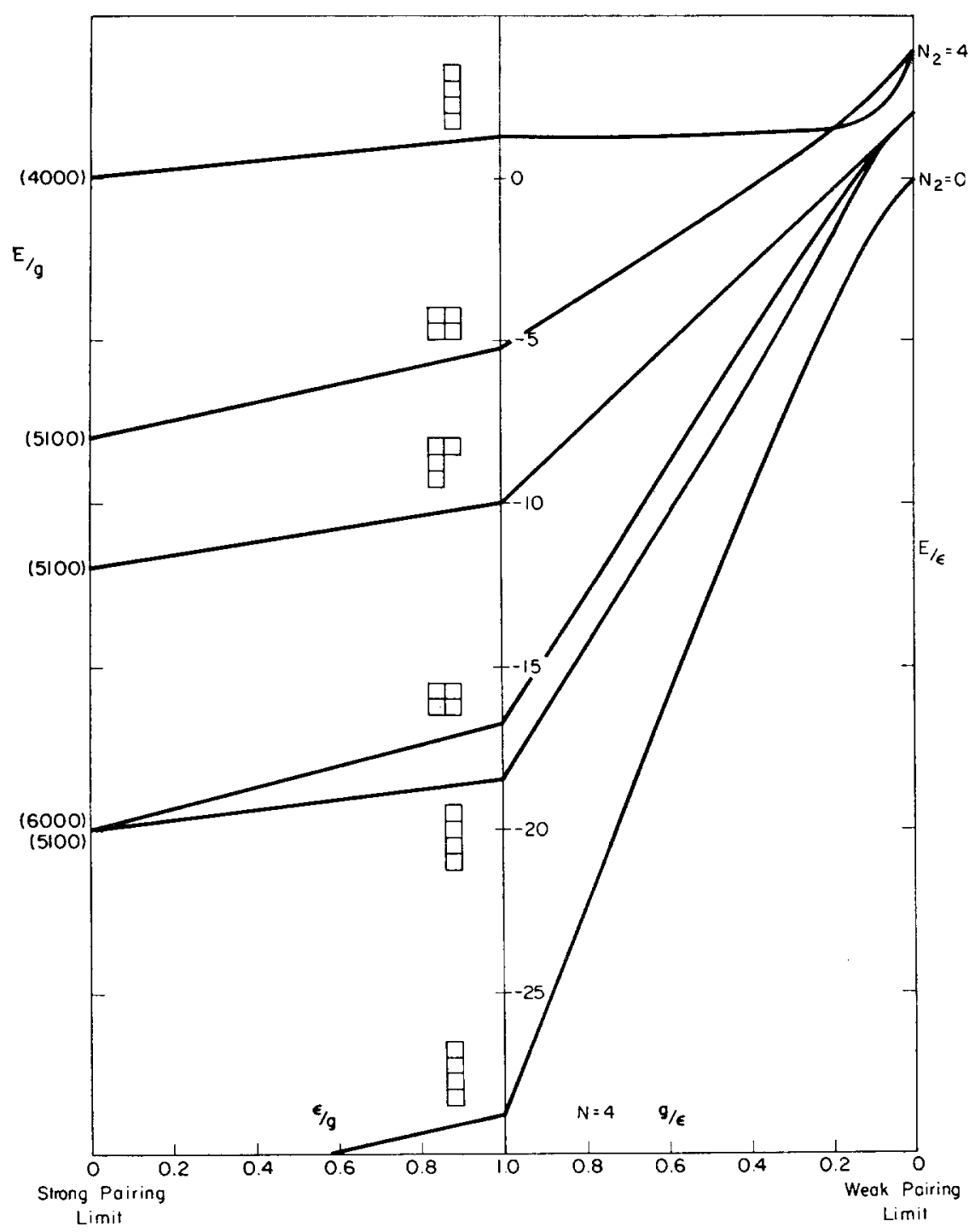

Fig. 1. $N=4$ : Pairing energy spectrum for four nucleons distributed over a spectrum of two singleparticle levels of $l=0\left(s\right.$ or $\left.\Omega_{1}=1\right)$ and $l=2\left(d\right.$ or $\left.\Omega_{2}=5\right)$-like character. Individual level seniorities are zero, $v_{i}=0$, for the two levels. The energy of the first (s-like) level is considered as zero, while the second level (d-like) is taken at energy $\varepsilon$ above the first level. The curves show the energies (in units of $g$ ) as a function of $\varepsilon / g$ on the left side to the limit of $g \rightarrow \infty$, in which all the nucleons are distributed in a degenerated s-d level $(\Omega=6)$. O(8) quantum numbers are used to denote the states in that extreme limit. The curves also show the energies (in units of $\varepsilon$ ) as a function of $g / \varepsilon$ on the right side to the limit of $g \rightarrow 0$, in which the $N$ nucleons are not interacting. The SU(4) Young tableau which is "a good quantum number" for all pairing strengths is shown for each level. For the scalar representation $U(4)$ is shown. 
sults are shown in figs. 1-4 where the energies are plotted for different values of the parameter $\frac{\varepsilon}{g}$. For $\frac{\varepsilon}{g}<1$ the energies are plotted in units of $g$ versus $\frac{\varepsilon}{g}$ on the left side;

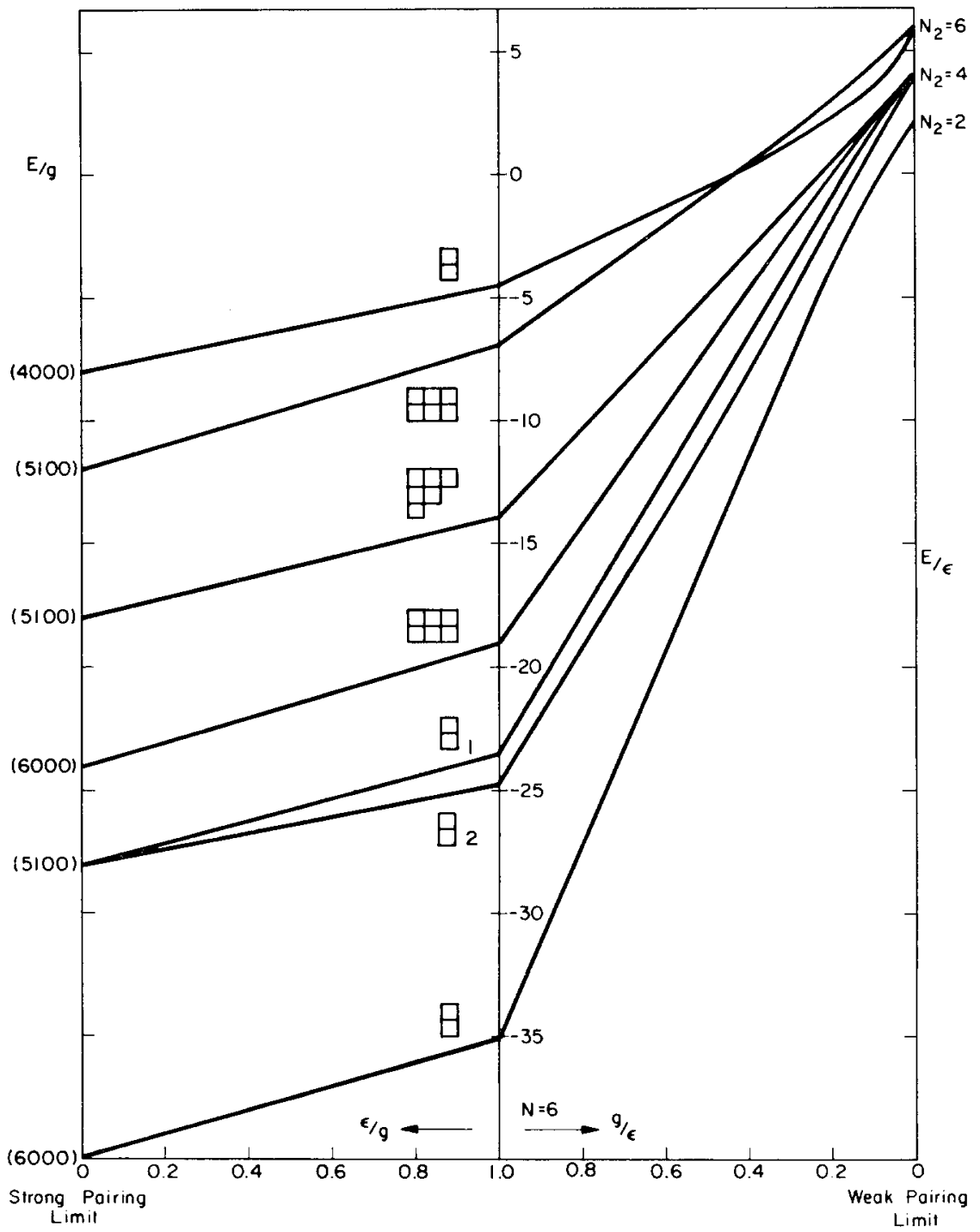

Fig. 2. $N=6$ : Pairing energy spectrum for $N=6, \Omega_{\mathrm{t}}=1, \Omega_{2}=5, v_{i}=0$. The notation is the same as that for fig. 1 .

on the other hand, for $\frac{\varepsilon}{\theta}>1$, the energies are plotted in units of $\varepsilon$ versus $\frac{g}{\varepsilon}$ on the right side of the figures.

On the extreme right, that is with $\frac{g}{\varepsilon}=0$, the extreme limit of weak pairing is reached; and the energies are degenerated to the single-particle energies, which depend 
only on the number of particles in the second level. When the pairing strength is "turned on," the levels are split. Since all $g_{\alpha \beta}^{i j}$ are assumed to have one common value in this calculation, the total Wigner supermultiplet is a good quantum number

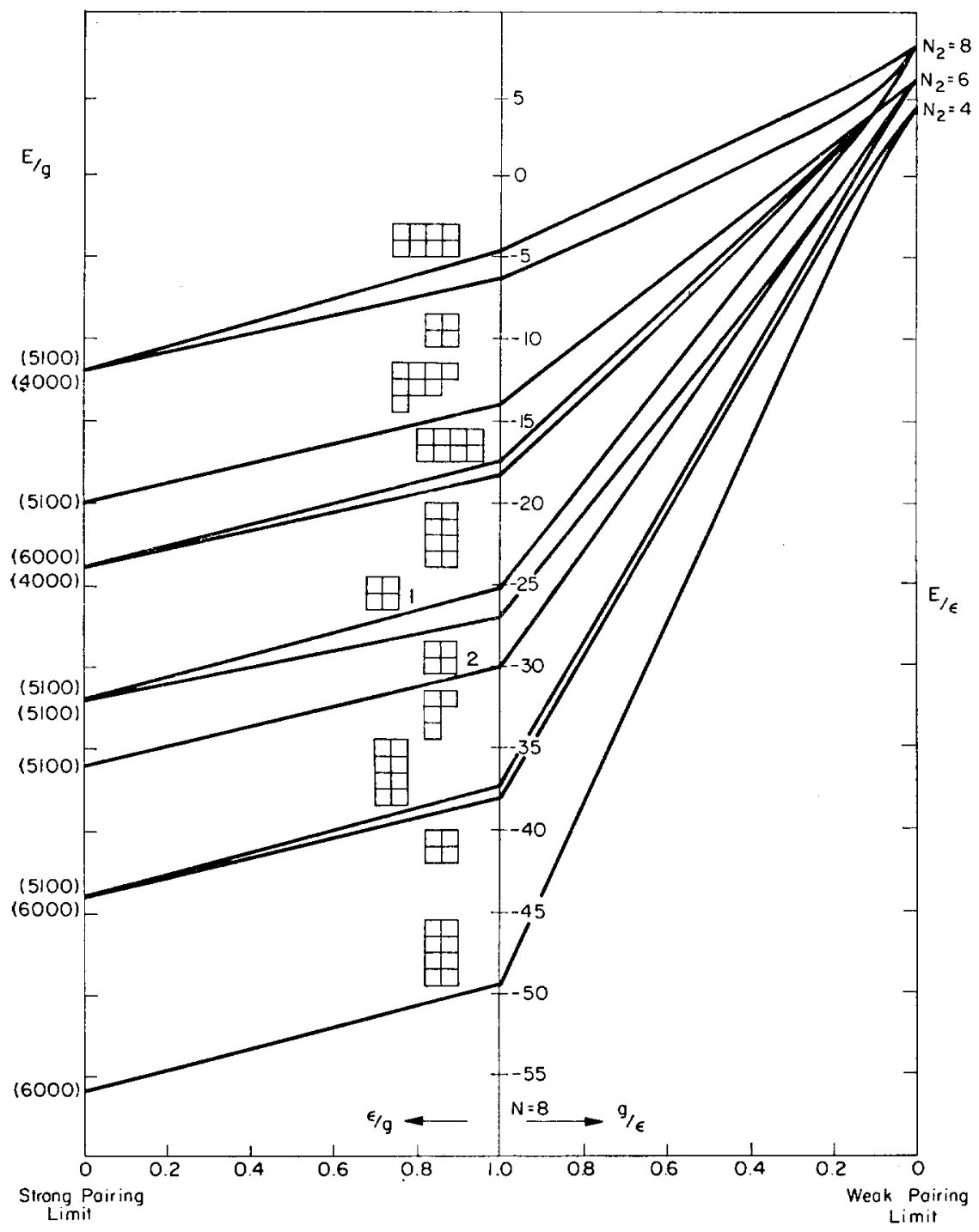

Fig. 3. $N=8$ : Pairing energy spectrum for $N=8, \Omega_{1}=1, \Omega_{2}=5, v_{i}=0$. The notation is the same as that for fig. 1 .

for all values of $\frac{\varepsilon}{g}$. Levels with different total $S$ and $T$ belonging to the same Wigner supermultiplet are degenerate, and only the supermultiplet labels are indicated. When the pairing strength is turned on to such a great magnitude that $\frac{\varepsilon}{g}$ approaches zero, all 
single-particle effects disappear and the extreme pairing limit of a single level with $\Omega=\Omega_{1}+\Omega_{2}=6$ is reached.

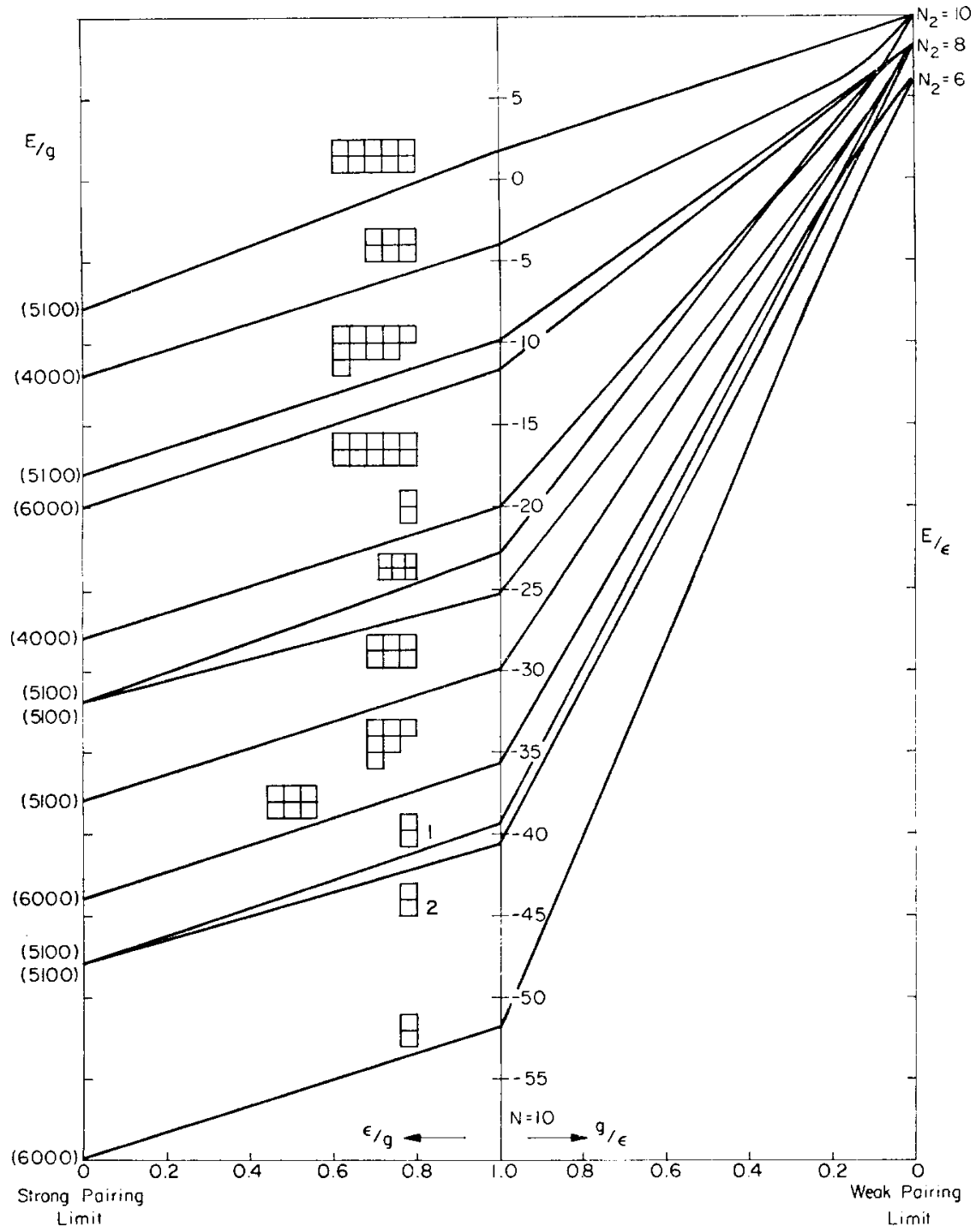

Fig. 4. $N=10$ : Pairing energy spectrum for $N=10, \Omega_{1}=1, \Omega_{2}=5 . v_{i}=0$. The notation is the same as that for fig. 1 .

In this extreme pairing limit, the energies are completely specified by the overall $O(8)$ representation labels and the total Wigner supermultiplet quantum numbers, and in terms of these are given by eq. (4.12) with $\Omega=6$. In order to understand this extreme pairing limit, the coupling of the simple $\mathrm{O}(8)$ representations corresponding 
to $v=0$ states for levels with $\Omega_{1}=1$ and $\Omega_{2}=5$ must be worked out. The result gives the coupling rule

$$
(50000) \times(1000)=(6000)+(5100)+(4000) .
$$

In the extreme pairing limit, $\frac{\varepsilon}{g}=0$, therefore, the energies are completely specified by the overall $O(8)$ representations $(6000),(5100)$ or $(4000)$ and the total Wigner supermultiplets (or $\mathrm{O}(6)$ representations) contained in these. The decomposition of $(\Omega 000)$ to representations of $\mathrm{O}(6)$ and nucleon number $N$ is given in sect. 3 . For the $O(8)$ representation $(5100)$ this decomposition is shown explicitly in table 6 . Certain of the levels show a two-fold Wigners supermultiplet degeneracy. These include all the two-fold cases for the $O(8)$ representation $(5100)$ listed in table 6 , as well as some additional accidental degeneracies such as that between (6000) [220], and (5100) [000] for $N=4$ (fig. 1), which can be seen from the strong pairing energy formula $(4.12)$.

TABLE 6

Branching rule of $(5100) \rightarrow N, \mathrm{O}(6)$

\begin{tabular}{|c|c|c|c|c|c|c|c|}
\hline \multicolumn{2}{|r|}{$N$} & \multicolumn{6}{|c|}{$\mathrm{O}(6)$ representations } \\
\hline 0 & (24) & $(000)$ & & & & & \\
\hline 2 & $(22)$ & $(100)$ & & & & & \\
\hline 4 & (20) & $(200)$ & $(110)$ & $(000)$ & & & \\
\hline 6 & (18) & $(300)$ & (210) & $(100)^{2}$ & & & \\
\hline 8 & (16) & $(400)$ & $(310)$ & $(200)^{2}$ & $(110)$ & $(000)$ & \\
\hline 10 & (14) & $(500)$ & $(410)$ & $(300)^{2}$ & $(210)$ & $(100)^{2}$ & \\
\hline 12 & & $(510)$ & $(400)^{2}$ & (310) & $(200)^{2}$ & $(110)$ & $(000)^{2}$ \\
\hline
\end{tabular}

In the intermediate coupling case, the total Wigner supermultiplet is a "good quantum number," and it is interesting to see how the various possible total Wigner supermultiplets for a given total $N$ can be obtained from the coupling of the level 1 supermultiplet $\left(n_{1} 00\right)$ with the level 2 supermultiplet $\left(n_{2} 00\right)$. This is illustrated in fig. 5 for the case $N=10=\left(N_{1}=2\right)+\left(N_{2}=8\right)$.

From the overall results illustrated by figs. $1-4$, some general conclusions may be drawn.

(i) The pairing effect is predominant for all but the very smallest values of $\frac{g}{\varepsilon}$. Except for a small splitting of the levels which are degenerate in the extreme strong pairing limit, the spectra in all cases are qualitatively very similar to the strong pairing limit down to a value of $\frac{g}{\varepsilon} \approx 0.2$. That is, with the exception of the extreme weak pairing limit, the pairing effects win out over the effects arising from single-particle excitations.

(ii) The pairing interaction is most effective in those Wigner supermultiplet states which are built from the largest possible number of columns of four. These columns of four have the general symmetry and $S=T=0$ character of an $\alpha$-cluster. The pairing interaction thus seems to favour states built from such clusters in that it tends 
to make such states more stable. For example, for $N=10$, the states with $N_{2}=10$ in the weak pairing limit are split such that the supermultiplet [3322] $=[110]$ is depressed much more than [4411] $=$ [330] which in turn lies below [5500], and the supermultiplet [3322] $=[110]$, is depressed to an energy level lower than some of the energy levels which grow out of $N_{2}=8$ after the pairing strength is increased only to $\frac{g}{\varepsilon}=0.1$. For the levels which grow out of the weak pairing limit $N_{2}=8$, a similar rule can be seen to hold, so that the energy levels corresponding to states in the first row of fig. 5 lie bigher than those for the 2 nd row, while those of the $3 \mathrm{rd}$ row built from states with two columns of four in level 2 are depressed to the lowest energies.

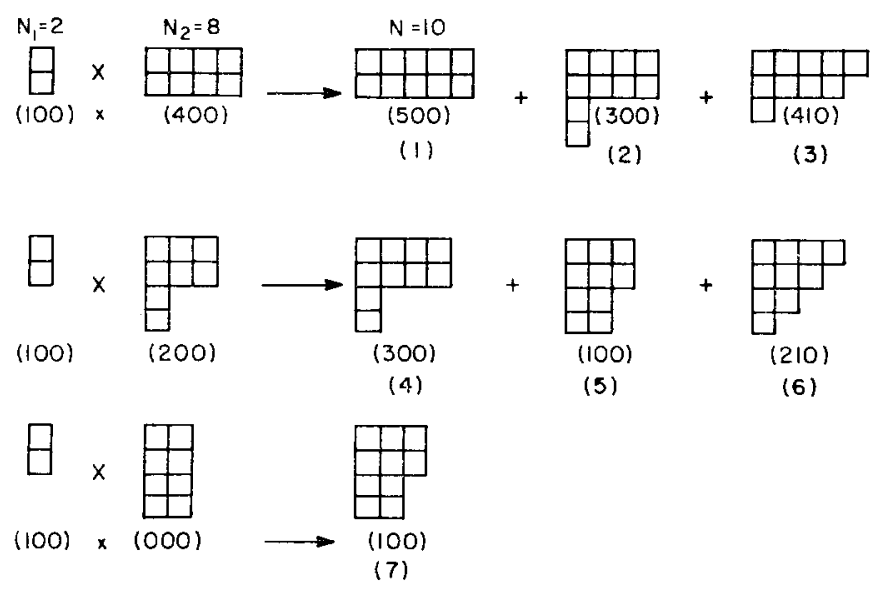

Fig. 5. The possible $U(4)$ representations for $N=10$ obtained from the coupling of seniority zero multiplets with $N_{1}=2$ and $N_{2}=8$.

Within the first row the ordering of energies, highest to lowest, is (3) > (1) > (2); that is the state with one column of four is lowest. Similarly, the energy ordering is (6) $>(4)>(5)$; and the overall ordering of those levels which grow out of the weak pairing limit $N_{2}=8$ is given by (3) $>(1)>(2)>(6)>(4)>(5)>(7)$ in the notation of fig. 5 .

Although the numerical calculations presented here have not been extensive, the calculations which have been carried out do seem to indicate two general results. The pairing interaction is very effective compared with the particle excitation energy, and pairing effects win out in the competition with single-particle excitations. Secondly the pairing interaction tends to make more stable those states built from the largest possible number of $\alpha$-like groupings of four particles, a "fouring" effect, which is not found and could not be understood in terms of a charge-independent pairing interaction in the $j-j$ coupling scheme.

The author is grateful to Professor K. T. Hecht for suggesting the problem, and his generous guidance through all the stages of this work. The author also wishes to thank Dr. Paul J. Ellis for his help with the computer programming. 


\section{References}

1) A. K. Kerman, Ann. of Phys. 12 (1961)

2) K. Helmers, Nucl. Phys. 23 (1961) 594

3) B. H. Flowers, and S. Szpikowski, Proc. Phys. Soc. 84 (1964) 193; 84 (1964) 673

4) J. C. Parikh, Nucl. Phys. 63 (1965) 214;

M. Ichimura, Progr. Theor. Phys. (Kyoto) 32 (1964) 757; 33 (1965) 215;

H. J. Lipkin, Lie groups for pedestrians (North-Holland Publ. Co., Amsterdam, 1965);

J. N. Ginocchio, Nucl. Phys. 74 (1965) 32I;

K. T. Hecht, Phys. Rev. 139 (1965) B794; Nucl. Phys. 102 (1967) 11

5) R. W. Richardson, Phys. Rev. 159 (1967) 792, also part II, to be published

6) I. M. Gelfand, R. A. Minlos and Z. Ya. Shapiro, Representations of the rotation and Lorentz groups and their application (The Macmillan Company, New York, 1963) p. 353;

S. C. Pang and K. T. Hecht, J. Math. Phys. 8 (1967) 1233

7) M. Moshinsky and J. G. Nagel, Phys. Lett. 5 (1963) 173

8) K. T. Hecht and S. C. Pang, J. Math. Phys., in press

9) S. C. Pang, Univ. of Michigan, technical report (November, 1967) 